\title{
The WaZP galaxy cluster sample of the dark energy survey year 1
}

\author{
M. Aguena ${ }^{\circledR},{ }^{1,2 \star}$ C. Benoist,,${ }^{1,3}$ L. N. da Costa, ${ }^{1,4}$ R. L. C. Ogando ${ }^{\circledR}, 1,4$ J. Gschwend, ${ }^{1,4}$ \\ H. B. Sampaio-Santos, ${ }^{1,4}$ M. Lima, ${ }^{1,5}$ M. A. G. Maia,,${ }^{1,4}$ S. Allam, ${ }^{6}$ S. Avila, ${ }^{7}$ D. Bacon, ${ }^{8}$ E. Bertin,,${ }^{9,10}$ \\ S. Bhargava, ${ }^{11}$ D. Brooks, ${ }^{12}$ A. Carnero Rosell,${ }^{13,14}$ M. Carrasco Kind,${ }^{15,16}$ J. Carretero, ${ }^{17}$ M. Costanzi,${ }^{18,19}$ \\ J. De Vicente, ${ }^{20}$ S. Desai, ${ }^{21}$ H. T. Diehl,${ }^{6}$ P. Doel, ${ }^{12}$ S. Everett, ${ }^{22}$ A. E. Evrard ${ }^{23,24}$ I. Ferrero, ${ }^{25}$ A. Ferté, ${ }^{26}$ \\ B. Flaugher, ${ }^{6}$ P. Fosalba, ${ }^{27,28}$ J. Frieman, ${ }^{6,29}$ J. García-Bellido, ${ }^{7}$ P. Giles, ${ }^{11}$ R. A. Gruendl, ${ }^{15,16}$ \\ G. Gutierrez, ${ }^{6}$ S. R. Hinton, ${ }^{30}$ D. L. Hollowood, ${ }^{22}$ K. Honscheid, ${ }^{31,32}$ D. J. James, ${ }^{33}$ T. Jeltema, ${ }^{22}$ \\ K. Kuehn, ${ }^{34,35}$ N. Kuropatkin, ${ }^{6}$ O. Lahav, ${ }^{12}$ P. Melchior,${ }^{36}$ R. Miquel, ${ }^{17,37}$ R. Morgan, ${ }^{38}$ A. Palmese, ${ }^{6,29}$ \\ F. Paz-Chinchón, ${ }^{16,39}$ A. A. Plazas, ${ }^{36}$ A. K. Romer, ${ }^{11}$ E. Sanchez, ${ }^{20}$ B. Santiago, ${ }^{1,40}$ M. Schubnell, ${ }^{24}$ \\ S. Serrano, ${ }^{27,28}$ I. Sevilla-Noarbe,${ }^{20}$ M. Smith, ${ }^{41}$ M. Soares-Santos,${ }^{24}$ E. Suchyta,,${ }^{42}$ G. Tarle,${ }^{24}$ C. To, ${ }^{43}$ \\ D. L. Tucker ${ }^{6}$ and R. D. Wilkinson ${ }^{11}$
}

Affiliations are listed at the end of the paper

Accepted 2021 January 26. Received 2021 January 22; in original form 2020 August 21

\begin{abstract}
We present a new (2+1)D galaxy cluster finder based on photometric redshifts called Wavelet Z Photometric (WaZP) applied to DES first year (Y1A1) data. The results are compared to clusters detected by the South Pole Telescope (SPT) survey and the redMaPPer cluster finder, the latter based on the same photometric data. WaZP searches for clusters in wavelet-based density maps of galaxies selected in photometric redshift space without any assumption on the cluster galaxy populations. The comparison to other cluster samples was performed with a matching algorithm based on angular proximity and redshift difference of the clusters. It led to the development of a new approach to match two optical cluster samples, following an iterative approach to minimize incorrect associations. The WaZP cluster finder applied to DES Y1A1 galaxy survey (1511.13 deg ${ }^{2}$ up to $m_{i}=23 \mathrm{mag}$ ) led to the detection of 60547 galaxy clusters with redshifts $0.05<z<0.9$ and richness $N_{\text {gals }} \geq 5$. Considering the overlapping regions and redshift ranges between the DES Y1A1 and SPT cluster surveys, all SZ based SPT clusters are recovered by the WaZP sample. The comparison between WaZP and redMaPPer cluster samples showed an excellent overall agreement for clusters with richness $N_{\text {gals }}$ ( $\lambda$ for redMaPPer) greater than 25 (20), with 95 per cent recovery on both directions. Based on the cluster cross-match, we explore the relative fragmentation of the two cluster samples and investigate the possible signatures of unmatched clusters.
\end{abstract}

Key words: methods: data analysis - surveys - galaxies: clusters: general-galaxies: distances and redshifts.

\section{INTRODUCTION}

The abundance and clustering properties of galaxy clusters have been shown to be powerful probes to constrain cosmological models, provided that their astrophysical properties are well characterized and linked to theoretical predictions (e.g. Lima \& Hu 2005; Vikhlinin et al. 2009; Mantz et al. 2010; Benson et al. 2013; Weinberg et al. 2013; Planck Collaboration XIII 2016; DES Collaboration et al. 2020).

Galaxy clusters can be detected from X-ray observations (Kim et al. 2007; Lloyd-Davies et al. 2011; Adami et al. 2018) and from the Sunyaev-Zel'dovich (SZ) effect (Bleem et al. 2015), but on-going and future large photometric surveys constitute a very promising approach to build large controlled galaxy cluster samples for both

^E-mail: aguena@linea.gov.br cosmological and astrophysical studies. These include the Kilo Degree Survey (KIDS; de Jong et al. 2013), the Dark Energy Survey (DES; The Dark Energy Survey Collaboration Flaugher 2005), PanSTARRS (Kaiser et al. 2002), the Legacy Survey of Space and Time (LSST; LSST Science Collaboration 2009), and the European Space Agency Cosmic Vision mission (Euclid; Laureijs et al. 2011).

However, detecting and characterizing clusters through their galaxy component remains a non-trivial task, especially when considering lower mass or higher redshift clusters. One has to distinguish between gravitationally bound groups of galaxies and projection effects due to the underlying large-scale distribution of galaxies. Projection effects not only impact detection, but also several fundamental properties of detected clusters, such as centring, redshift, and mass proxy (e.g. cluster richness).

Many automated algorithms were developed in the last three decades to overcome these difficulties. Automatic optical cluster finders can generally be described as algorithms searching for cluster 
scale galaxy overdensities. Galaxies are first filtered (or weighted) following prescriptions to increase the detection contrast relative to background galaxies. The main techniques used for searching galaxy overdensities include kernel smoothing (e.g. Shectman 1985; Lumsden et al. 1992; Gladders \& Yee 2000; Adami et al. 2010), friends-of-friends (e.g. Botzler et al. 2004; Trevese et al. 2007; Wen, Han \& Liu 2012), or Voronoi tesselation (e.g. Ramella et al. 1999; Soares-Santos et al. 2011). These techniques have been applied to galaxy catalogues that are usually previously filtered in one or several dimensions (e.g. magnitudes, colours, or photometric redshifts). More sophisticated approaches assume an underlying cluster model (e.g. density profile, luminosity function, colour content) and identify clusters in likelihood maps based on matched filter techniques (e.g. Postman et al. 1996; Olsen et al. 1999; Koester et al. 2007; Olsen et al. 2008; Rykoff et al. 2014, 2016; Bellagamba et al. 2018).

A typical assumption of optical cluster finders is to consider the presence of a red sequence of galaxies (Gladders \& Yee 2000; Koester et al. 2007; Hao et al. 2010; Murphy, Geach \& Bower 2012). In low redshift clusters, the most luminous galaxies define a tight sequence in the colour-magnitude diagram, the so-called 'E/S0 ridge line', or 'red sequence'. Red sequence galaxies have very uniform colours and are among the reddest galaxies at a given redshift. Because of the strong $4000 \AA$ break in their rest-frame spectra, their colour is tightly correlated with redshift and can be used to estimate cluster redshifts. This feature has been observed in rich clusters up to $z \sim$ 1.7 (e.g. Mei et al. 2009; George et al. 2011; Wetzel et al. 2013; Strazzullo et al. 2019). However, some galaxy clusters observed at high redshifts can display appreciable star formation, even in cluster cores (e.g. Brodwin et al. 2013), weakening the red sequence.

Cluster finders such as maxBCG (Koester et al. 2007), redMaPPer (Rykoff et al. 2014, 2016), or RedGOLD (Licitra et al. 2016) rely on the red sequence for cluster detection and redshift estimate. In the context of recent surveys, cluster finders not based on the red sequence usually rely on photometric redshifts. An alternative based on the knee of the cluster luminosity function was also used in the context of surveys with a limited number of passbands (e.g. Postman et al. 1996; Olsen et al. 1999).

Even if current automated optical cluster finders are all able to identify rich clusters, evaluating their performances over broad ranges of masses and redshifts and deriving the selection function of the resulting cluster samples remain highly complex tasks. On the theoretical side, this requires the development of ever more realistic simulated galaxy catalogues. On the observational side, we need multiple surveys covering the same area at different frequency domains to detect clusters through a variety of signatures.

There is not a unique methodological framework to evaluate and compare the performances of optical cluster finders. A variety of approaches have been proposed, based either on mock galaxy catalogues (e.g. Euclid Collaboration 2019 and references therein), or on real data, or even on a mix of the two approaches (e.g. Goto et al. 2002; Kim et al. 2002; Rykoff et al. 2014; Costanzi, M. \& Rozo, E. et al. 2019).

Within simulations assumptions, simulation-driven methods provide a truth table useful for comparison, with clusters embedded in realistic large-scale structures. These methods also offer a direct link between galaxy clusters and dark matter haloes. However, they rely on sophisticated modelling that so far does not fully reproduce all observed galaxy properties, specially at high redshift (e.g. DeRose et al. 2019). In addition to this fundamental problem, mock catalogues do not usually reproduce the variety and complexity of defects occurring in observed images and introduced at the stage of source extraction and classification. DES has recently started to deal with this using the BALROG algorithm (Suchyta et al. 2016), which embeds simulations into real data and should accompany future releases.

Addressing the cluster selection function based on real data is necessarily limited by the absence of an absolute reference to confront the results of any cluster finder. None the less, useful information can be extracted from the cross-match of a given optical cluster sample with detections based on different tracers that do not suffer from the same projection effects (e.g. Saro et al. 2015). A better understanding of the galaxy cluster selection function can also be improved from cross-matching samples from different optical cluster finders. The resulting samples may differ not only due the different adopted physical assumptions but also due to the details of cluster finder implementation (Ascaso et al. 2017; Aguena \& Lima 2018), or even the way the algorithms deal with specific features of real data (e.g. noise, missing data, star/galaxy separation, etc.).

DES has produced galaxy cluster samples with the redMaPPer algorithm which were published in Rykoff et al. (2016) and McClintock, T. \& Varga, T. N. et al. (2019), based on DES Science Verification and DES-Y1 data releases, respectively. These samples led to several studies focusing on the mass-richness relation (Melchior et al. 2015; Saro et al. 2015; Palmese et al. 2016; Zhang et al. 2016; Melchior, P. \& Gruen, D. et al. 2017; Saro et al. 2017; Pereira et al. 2018; McClintock, T. \& Varga, T. N. et al. 2019; Bleem et al. 2020; Pereira et al. 2020). Complementary analyses were also performed on cluster luminosity function (Zhang et al. 2017), baryon content (Chiu et al. 2018), and cluster mis-centring relative to X-ray detections (Zhang et al. 2019). These clusters were also used for the detection of voids (Pollina et al. 2019). Most of these studies contribute to the work on cosmological constraints using DES first year release redMaPPer clusters (DES Collaboration 2020).

In this paper, we present the Wavelet Z Photometric (WaZP) cluster finder and apply it to DES-Y1 data. WaZP is an optical cluster finder designed to detect clusters based mainly on the spatial clustering of galaxies using photometric redshift information. The primary motivation for developing WaZP is to limit assumptions on the properties of cluster galaxies such as the presence of a red sequence, the shape of their luminosity function, or radial profile, assumptions that may impact cluster detection, in particular at high redshift or at lower mass regime.

Here, the WaZP DES-Y1 sample is compared to cluster samples obtained from the SPT survey based on the SZ effect and those obtained by the redMaPPer cluster finder on the same DES-Y1 data set. The first comparison allows us to test how well the WAZP algorithm recovers the massive clusters detected by the SPT. The second comparison, for which the two samples have similar cluster densities, gives insights on the relative completenesses of the two optical cluster samples, and on the derived properties of the common detections. Variations may occur in the samples due to the different assumptions made in terms of cluster modelling. They may also occur due to different uses of the underlying galaxy data set as the WAZP algorithm uses magnitude information from all bands through photometric redshifts and $i$-band as a reference band, whereas redMaPPer uses combinations of band pairs (colours) to select likely red sequence galaxies and $z$-band as a reference. Considered survey coverage can therefore be slightly different with one approach or the other. Depth variability in all bands will also impact differently cluster detection with each algorithm. While the comparisons performed here provide a valuable heuristic approach to partly qualify the cluster samples, a complete evaluation of the WaZP sample requires to address a quantitative assessment of its 
purity, a work that will be presented in a companion paper based on mock galaxy catalogues.

This paper is organized as follows. In Section 2, we describe the DES Y1 data used in our analysis. In Section 3, we describe the main properties of the WaZP cluster finder. In Section 4, we present our main results on DES-Y1 data. In Section 5, we matched the derived WaZP cluster samples to the SZ sample and to the redMaPPer sample obtained from DES-Y1 data. Finally, in Section 6 we analyse the properties of the catalogue, and discuss the differences between our catalogue and the others compared in Section 5.

Throughout this work, we fix cosmological parameters from the Planck results (Planck Collaboration XIII 2016) for a flat $\Lambda \mathrm{CDM}$ model with $\Omega_{m}=0.308$ and $H_{0}=67.8 \mathrm{~km} \mathrm{~s}^{-1} \mathrm{Mpc}^{-1}$.

\section{DATA}

The DES is an imaging survey covering $5000 \mathrm{deg}^{2}$ in 5 bands $(g$, $r, i, z, Y$ ) (e.g. Flaugher 2005; Dark Energy Survey Collaboration 2016; Diehl et al. 2016). In this paper, we use the DES Year 1 data release, which has been extensively studied by the DES collaboration (e.g. Shipp et al. 2018; Troxel et al. 2018). The DES built the Dark Energy Camera (DECam; Flaugher et al. 2015) with a field of view diameter of 2.2 deg covered by 520 Megapixels distributed on a mosaic of $62 \mathrm{CCDs}$ that are extra sensitive on the red part of the electromagnetic spectrum, enhancing its capability of observing high redshift galaxies. DECam is installed on CTIO 4-m Blanco telescope prime focus, and its observations follow a strategy that optimizes pointings based on properties like weather and moon phase (Neilsen et al. 2019). The images are reduced and calibrated by the DES Data Management (DESDM) team at the National Center for Supercomputing Applications (NCSA). The DESDM pipeline includes the reduction of single-exposure images, their co-addition into deeper images, source extraction, and calibration, all resulting in the creation of the main scientific catalogue (Morganson et al. 2018).

The DES Year 1 Annual Release (Y1A1; Abbott et al. 2018) co-added catalogue used in this analysis covers a total area of $\sim 1520 \mathrm{deg}^{2}$, split into two main wide regions. One of them has an area of $\sim 140 \mathrm{deg}^{2}$ overlapping the SDSS Stripe 82 area (Aihara et al. 2011). The other part has an area of $\sim 1380 \mathrm{deg}^{2}$ overlapping the South Pole Telescope footprint (SPT; Carlstrom et al. 2011). In the following, we will refer to these two regions as Y1-S82 and Y1SPT, respectively. They were observed with three to four exposures in each filter (Drlica-Wagner et al. 2018).

In addition to catalogues, we also use ancillary maps to track defects and foreground objects (e.g. bright stars, very bright galaxies, and globular clusters) all over DES footprint. The resulting coverage map is represented by a detection fraction map, where pixels have values of area fraction from 0 to 1 . We also use systematic maps to track observing conditions across the footprint, such as number of exposures, seeing, and airmass (Leistedt et al. 2016). These maps are combined to produce depth maps based on galaxy magnitude limits, as described in Rykoff, Rozo \& Keisler (2015). All maps are recorded in HEALPIX format (NSIDE $=4096)$ (Górski et al. 2005).

The Y1A1 co-add catalogue and maps produced by DES DM were transferred to the Laboratório Interinstitucional de e-Astronomia (LIneA $)^{1}$ and ingested into the data base associated to the DES Science Portal (henceforth, the Portal) as described in Fausti Neto et al. (2018). We used the Portal infrastructure to create a galaxy Value Added Catalogue (VAC) tailored for galaxy cluster search

${ }^{1}$ http://www.linea.gov.br based on photometric redshift. The creation of the galaxy VAC includes: computation of photometric redshifts, star-galaxy classification, and pruning regions and objects to produce a clean galaxy catalogue with well controlled levels of completeness and homogeneity. Along with the VAC, a final footprint map in HEALPIX format is created, reflecting the selection and pruning applied to this VAC.

The computation of photometric redshifts relies on the machinelearning algorithm DNF (Directional Neighbourhood Fitting; De Vicente, Sánchez \& Sevilla-Noarbe 2016), operated in Euclidean Neighbourhood Fitting (ENF) mode since tests using DES Y1 data have shown that ENF mode is considerably faster, while providing similar results as in DNF mode. DNF uses as input observables SEXTRACTOR MAG_AUTO magnitudes (Bertin \& Arnouts 1996). DNF was trained with a large sample of spectroscopic redshifts extracted from a compilation of 29 public surveys intercepting the DES footprint. Quality flags of all surveys are brought to a common standard following OzDES approach (Yuan et al. 2015). As described in Gschwend et al. (2018), sources with flags 0 and 1 have unknown redshift, flag 2 redshifts are not reliable, flag 3 redshift reliability is above 90 per cent confidence, and flag 4 is attributed to a trusted redshift (over 99 per cent confidence). The DES photometric catalogue is matched to this spectroscopic redshift sample with a 1.0 arcsec search radius and down to $m_{i}=23 \mathrm{mag}$, producing a catalogue of 101971 galaxies with a mean redshift of 0.63 , and covering the redshift range $z=0-1.1$. Although colour and magnitude distributions of this spectroscopic sample differ from the global photometric set under study, we stress that it does cover the same colour-magnitude ranges with the exception of faint low redshift galaxies (typically magnitudes fainter than 19 and redshifts below 0.15$)$. The spectrophotometric catalogue is then randomly split into a training and a validation sets. Details about all the steps carried out to compute photometric redshifts in the DES Science Portal for Y1A1 data are described in Gschwend et al. (2018).

Star-galaxy classification follows a morphological prescription developed within the DES consortium called MODEST, described by equations (3) and (4) of Sevilla-Noarbe et al. (2018). It mainly depends on the SEXTRACTOR SPREAD_MODEL (Desai et al. 2012; Bouy et al. 2013) and its error, assessing how extended is the source to the local PSF. Note that this classification is based on the DES $i$-band and extends to the faintest sources.

Based on bad regions maps (Drlica-Wagner et al. 2018), areas around bright stars, bleed trails, bright foreground galaxies, or globular clusters (from Harris 2010) are removed from the footprint. Pixels with an effective coverage $\leq 0.1$ are discarded from our analysis. We also exclude regions not covered simultaneously by $g, r, i, z$ bands with a minimum of 90 s total exposure time. Besides region-based filtering, we also discard individual sources based on SEXTRACTOR FLAGS (only sources with FLAGS $\leq 3$ are kept), apply a magnitude cut $\left(m_{i} \geq 15\right)$, and colour cuts $\left(-2.0 \leq m_{g}-m_{r} \leq 10.0\right.$; $\left.-2.0 \leq m_{r}-m_{i} \leq 4.0 ;-2.0 \leq m_{i}-m_{z} \leq 4.0\right)$.

Depth maps, defined here as $10 \sigma$ limiting magnitude maps, were built following the method described in Rykoff et al. (2015). These maps correspond to the limit where the flux is at least 10 times its variance $\sigma$, computed from the magnitude errors. This definition assures a galaxy completeness larger than 90 per cent. Fig. 1 shows what survey area fraction is covered at a given $10 \sigma i$-band limiting magnitude (the $i$-band being the reference band in this work). The whole survey is at least as deep as $m_{i}=22.27$ and shallower than $m_{i}$ $=23.25$ with half of the survey area reaching $m_{i}=22.7$. In Fig. 2 we compare galaxy number counts for Y1-S82 and Y1-SPT with number counts from Arnouts et al. (2001) and Capak et al. (2007) as 


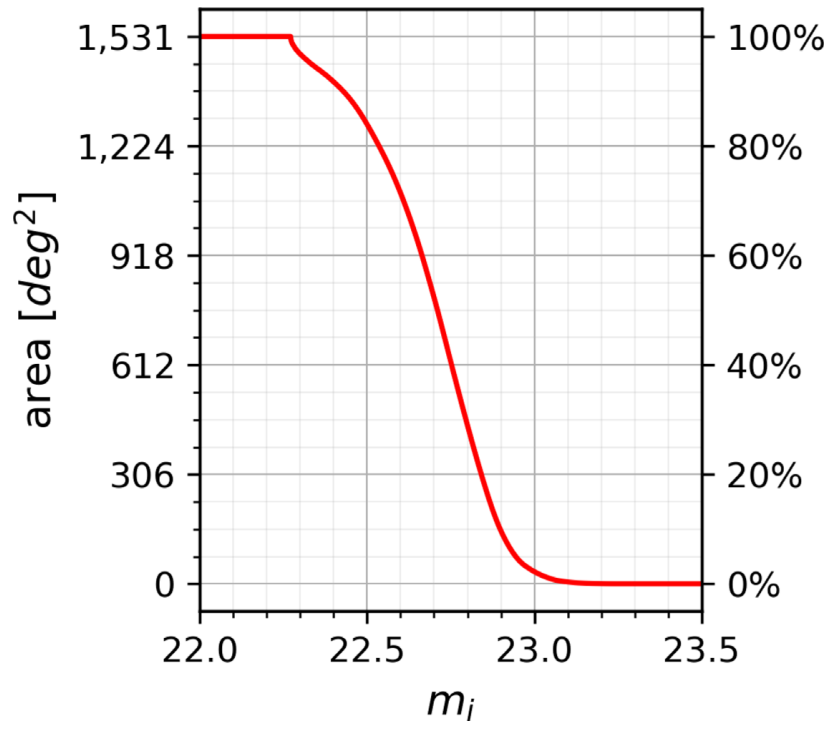

Figure 1. The effective area coverage of DES-Y1 as a function of the limiting magnitude in the $i$-band.

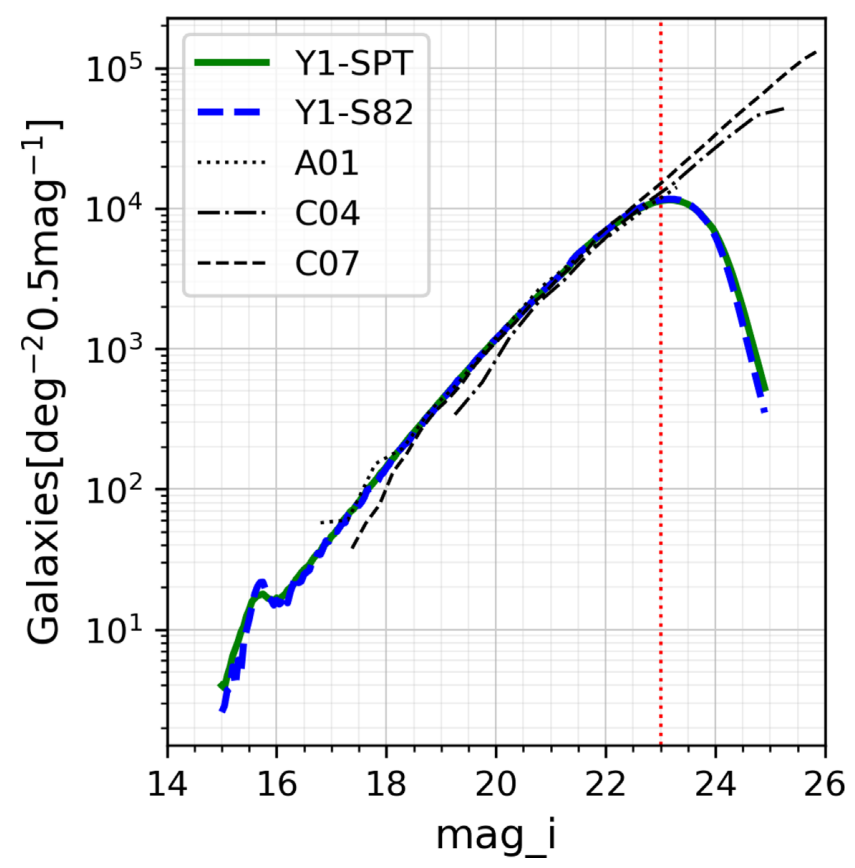

Figure 2. Galaxy density for Y1-S82 (thick blue solid line) and Y1-SPT (thick green dashed line) compared to Arnouts et al. 2001 (A01), Capak et al. 2004 (C04) and Capak et al. 2007 (C07). Magnitude limits of 22.5 and 23 mag are also noted as red vertical dotted and dashed line, respectively.

compiled by Nigel Metcalfe. ${ }^{2}$ The surveys used for comparison are deeper than our own, and number counts are comparable through a wide range of magnitudes up to $m_{i} \sim 22.5 \mathrm{mag}$, beyond which we observe an increasing deficit of galaxies, consistent with the median depth of the survey.

Based on the survey depth shown above, the present analysis considers galaxies down to a limiting magnitude $m_{i}=23$ ( 98 per cent of the survey area). The resulting galaxy VACs for Y1-S82 and
Table 1. VACs properties for both Y1-S82 and Y1-SPT.

\begin{tabular}{lcccc}
\hline Region & Galaxies & $\begin{array}{c}\text { Area } \\
\left(\mathrm{deg}^{2}\right)\end{array}$ & $\begin{array}{c}\text { Density } \\
\left(\mathrm{Gal} \mathrm{arcmin}^{-2}\right)\end{array}$ & $\begin{array}{c}\text { Mean } \\
\text { PHOTO-Z }\end{array}$ \\
\hline Y1-S82 & 4721380 & 143.66 & 9.13 & 0.65 \\
Y1-SPT & 45206403 & 1387.47 & 9.05 & 0.63 \\
\hline
\end{tabular}
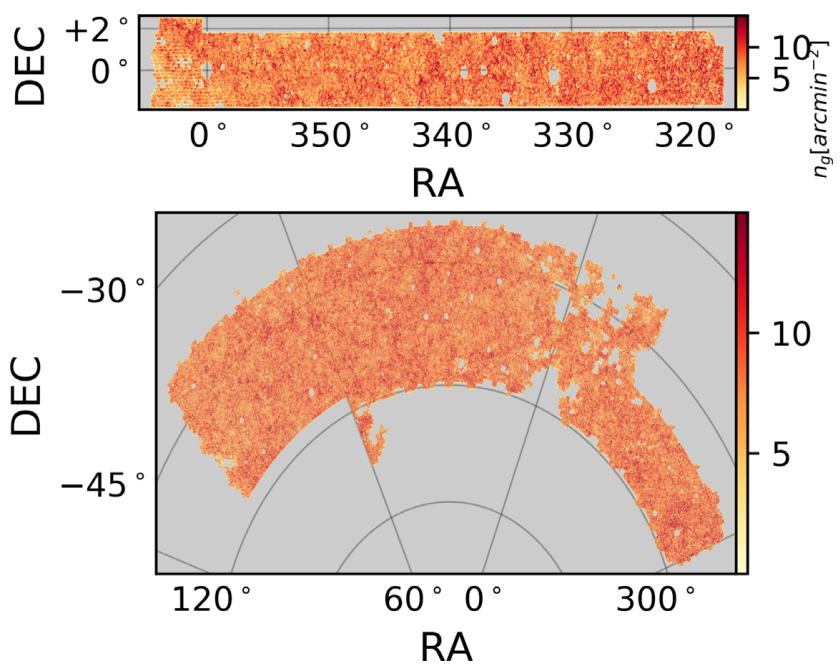

Figure 3. Spatial distribution of galaxies with $m_{i} \leq 23$ for Y1-S82 (top) and Y1-SPT (bottom). Colour bar displays density of galaxies per pixel at NSIDE $=1024\left(\approx 11 \operatorname{arcmin}^{2}\right)$.

Y1-SPT contain, respectively, 4721380 and 45206403 galaxies, in a total of 49927783 galaxies covering $1511.13 \mathrm{deg}^{2}$. Both regions have similar galaxy number densities and mean photometric redshift (Table 1).

Fig. 3 presents the projected galaxy distribution of Y1-S82 (top) and Y1-SPT (bottom). ${ }^{3}$ The distributions are fairly uniform and galaxy densities comparable. Holes caused by masking and dents on the footprint caused by unobserved regions can be seen in both regions.

Normalized photometric redshift distributions are shown in Fig. 4 for both regions. Our magnitude cut leads to a mean $z_{\text {phot }} \sim 0.63$ in both cases and very similar distributions. They both suffer from a counts drop around $z \sim 0.4$ due to the lack of the $u$-band. This can also be seen in the left-hand panel of Fig. 5 where photometric redshifts are compared to spectroscopic redshifts for a validation sample of 50476 galaxies built during DNF processing. There is an excellent correlation between spectroscopic and photometric redshifts; however, galaxies around $z_{\text {spec }} \sim 0.3$ show a very large scatter, especially towards higher values of $z_{\text {phot }}$. This can also be seen in the right-hand panel of Fig. 5, where we assessed the global quality of the photometric redshifts by characterizing the average bias and standard deviation of $\left(z_{\mathrm{ph}}-z_{\mathrm{sp}}\right) /\left(1+z_{\mathrm{sp}}\right)$. These points will be examined in detail in Sections 4 and 6.

\section{THE WAZP CLUSTER FINDER ALGORITHM}

The WaZP cluster finder is designed to detect galaxy clusters from multiwavelength optical imaging galaxy surveys. It searches for projected galaxy overdensities in photometric redshift space 


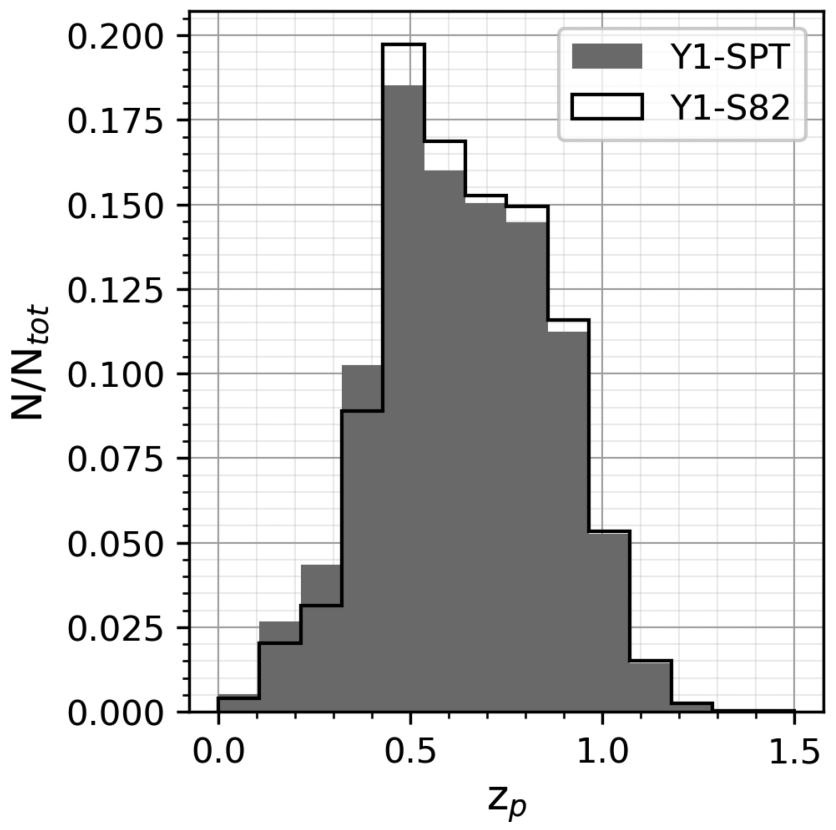

Figure 4. Normalized distribution of photometric redshifts for galaxies in Y1-S82 (dashed line) and Y1-SPT (solid line).

without any assumption on the red sequence. In a nutshell, WaZP first slices the galaxy catalogue in photometric redshift space, and then generates smooth wavelet-based density maps for each slice where peaks are extracted (see Fig. 6). These overdensity peaks are then merged to create a unique list of clusters and associated galaxy members. Hereafter, these various steps are described in detail.

(i) Slicing in photometric redshifts. By photometric redshift slices, we mean here the photometric redshift support over which individual galaxy redshift PDF's are integrated around a given redshift of interest. Therefore, at a given considered redshift, galaxies are weighted by that quantity. These weights are used to build density maps at different redshifts or estimate richnesses as described in the next steps.

The adopted strategy to define photometric redshift slices is based on the statistical comparison of the 'best-estimate' discrete photometric redshifts (taken here as the mean of the galaxy redshift PDF) and corresponding spectroscopic redshifts if available. Based on available spectroscopic samples, the mean bias and scatter of photometric redshifts $\left(z_{\mathrm{ph}}\right)$ relative to spectroscopic redshifts $\left(z_{\mathrm{sp}}\right)$ were derived. Following the standard way to evaluate the performance of photometric redshifts (e.g. Ilbert et al. 2006), we computed statistics of $\left(z_{\mathrm{ph}}-z_{\mathrm{sp}}\right) /\left(1+z_{\mathrm{sp}}\right)$ as a function of both $z_{\mathrm{sp}}$ and $z_{\mathrm{ph}}$. The location and width of a slice is then built in such a way that it includes 95 per cent of the galaxies of a given spectroscopic redshift. The separation between two slices corresponds to a fourth of their width assuring a sufficient overlap to avoid missing clusters being between two consecutive slices.

(ii) Generation of galaxy number density maps. WaZP does not consider photometric redshifts as discrete values. Instead, it operates with redshift PDF's when provided, or generates them from the errors provided by the chosen photometric redshift algorithm. In each one of the slices defined above, galaxies are weighted by the integral of their redshift PDF over that slice. The resulting weighted RA-Dec distribution is then pixellized on a grid with a step of physical size 1/16th of an Mpc. This image is finally filtered using the wavelet task MR_FILTER from the multiresolution package MR/1 (Starck, Murtagh \& Bijaoui 1998). This task incorporates a statistically rigorous treatment of the Poisson noise, which allows us to keep significant structures in the desired scale range. Here we select structures with scales in the range $0.5-2 \mathrm{Mpc}$, typical of cluster scales, and apply a $3 \sigma$ iterative multiresolution thresholding with a B-spline wavelet transform.

(iii) Extraction of peaks. The smooth density maps obtained in the previous step are segmented, and in each object domain, one or more peaks are extracted. In the case of several peaks in one domain, depending on the distance between a peak and the closest saddle point, the peak can be merged or preserve its identity. Pixels of a domain are then distributed to peaks by proximity.

(iv) Assessing peak significance. The peak significance is chosen to be computed in a radius of $R_{S}=300 \mathrm{kpc}$, a radius that encloses typical cluster cores (Adami et al. 1998). To perform background statistics, the survey is pixellized with pixel areas equal to $\pi R_{S}^{2}$. Any pixel intersecting a bad region or an edge is removed. Standard counts in cells are then applied to estimate the mean density $\left(N_{\mathrm{bkg}}\right.$ - global $)$ and standard deviation $\left(\sigma_{\mathrm{bkg}}\right)$. The significance, defined as SNR $=$ $\left(N-N_{\text {bkg }}\right.$ - global $) / \sigma_{\text {bkg }}$, where $N$ is the total density of galaxies in a cylinder centred at the peak position, with a length that is the width of the redshift slice and an angular radius $R_{S}$.

(v) Peak merging along the redshift direction. As $z_{\mathrm{ph}}$ slices overlap, one can expect clusters to be detected in several consecutive slices. To build the final list of clusters, peaks of consecutive slices are associated, and only the slice in which the system has maximum significance is kept. Note that two clusters can be deblended along the line of sight if their distance in redshift is larger than $2 \times 3 \sigma_{\mathrm{dz} /(1+z)}$, where $\sigma_{\mathrm{dz} /(1+z)}$ denotes here the 68th percentile of the $\mathrm{dz} /(1+z)=$ $\left(z_{\mathrm{ph}}-z_{\mathrm{sp}}\right) /\left(1+z_{\mathrm{sp}}\right)$ distribution.

(vi) Centring and cluster redshift. The cluster centre is defined as the location of the density map peak. However, if the brightest cluster member is found within the first neighbouring pixels, then this galaxy marks the centre. This leads to a maximum shift of $100 \mathrm{kpc}$ from the peak location. Concerning the redshift, an initial value is derived as the mode of the sum of the galaxy redshift PDF's within a $0.5 \mathrm{Mpc}$ radius around the cluster centre. This value is refined iteratively based on the membership probabilities described below.

(vii) Assignment of membership probabilities. Membership probabilities $\left(P_{\mathrm{mem}}\right)$ are computed following the prescription given in Castignani \& Benoist (2016). In a nutshell, galaxies of the cluster field are piled up in a 3D grid (cluster-centric distance, magnitude, photometric redshift) where magnitudes and redshifts are included as probability distribution functions. The same is done for local background galaxies in (magnitude, photometric redshift) space. The local background galaxies are selected in a ring from 3 to $6 \mathrm{Mpc}$ to the cluster centre, whereas cluster field galaxies are selected within a $3 \mathrm{Mpc}$ disc. The membership probability is the combination of the probability to be at the cluster redshift and the probability not to be a background galaxy. The final membership probability at a given cluster-centric distance, magnitude, and redshift is derived from the density ratio between the cluster field and the background field. Note that, as in Castignani \& Benoist (2016), no parametric modelling is used for the radial density, nor for the luminosity function.

(viii) Richness and radius. The cluster richness and radius are estimated jointly. The richness is the sum of the membership probabilities within a radius that corresponds to an overdensity of 200 times the mean galaxy background number density (similar 

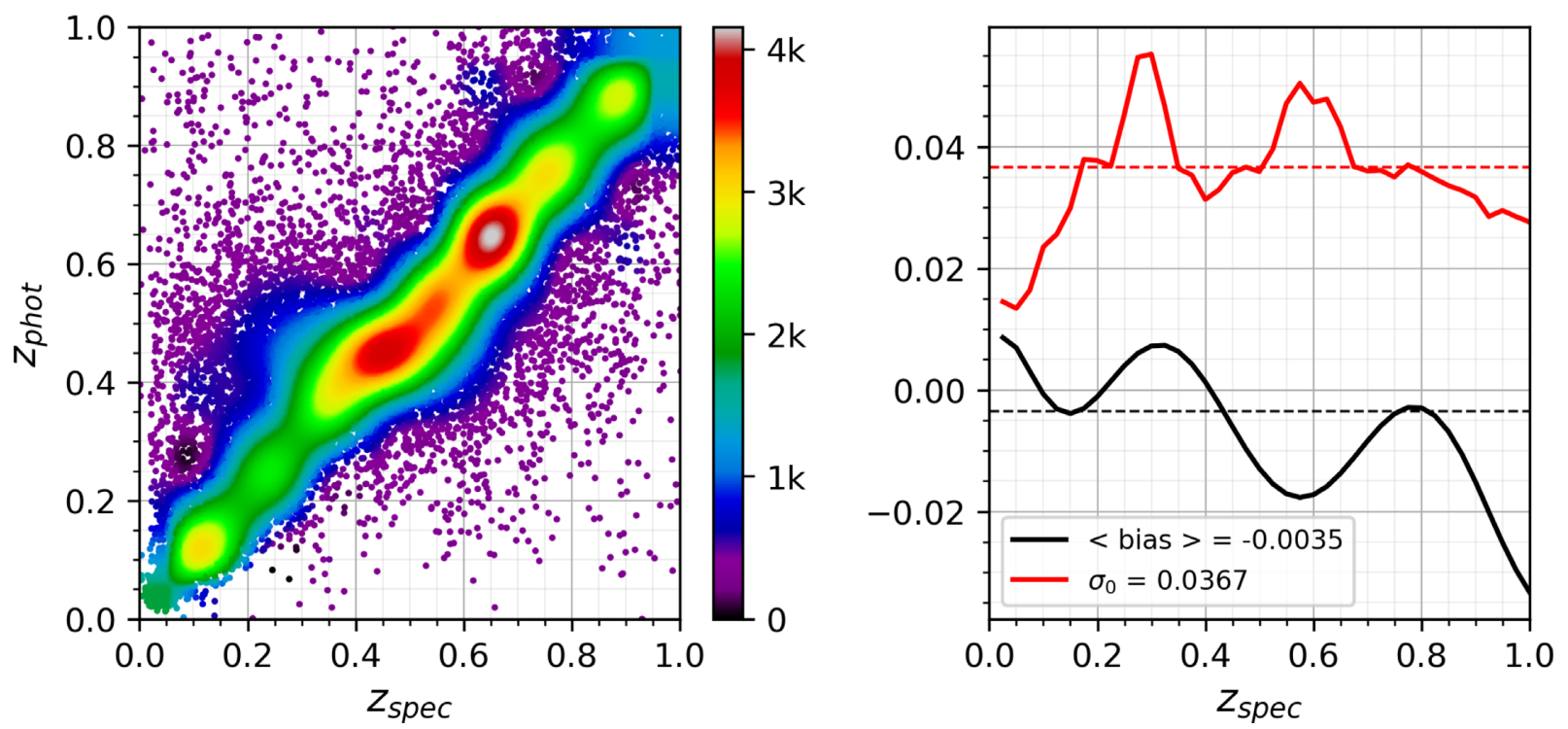

Figure 5. The DNF photometric redshifts compared to their associated spectroscopic redshifts for a validation sample of 50476 galaxies within the DES-Y1 footprint. The right-hand panel shows the bias and scatter of photometric redshifts relative to spectroscopic redshifts as a function of spectroscopic redshift.

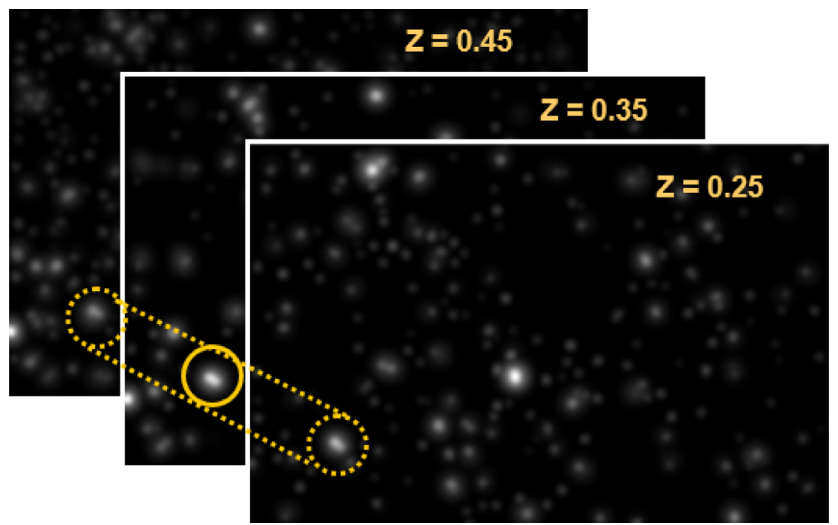

Figure 6. A schematic view of the WAZP algorithm. Here are shown the wavelet filtered galaxy density maps corresponding to three consecutive photometric redshift slices. It shows how a cluster can propagate along several slices.

to Hansen et al. 2005). This is done considering galaxies, both in the field and in the cluster, down to a given fraction of $L^{*}$ luminosity. Practically, galaxies brighter than $\mathrm{m}^{\star}\left(z_{\text {cluster }}\right)+\delta$ mag are counted, where $m^{\star}$ is the characteristic magnitude marking the knee of the luminosity function and $\delta$ mag is a fixed quantity, chosen here to be 1.5. The adopted definition allows us to produce 'redshift independent richnesses', in the sense that the same cluster seen at two different redshifts would have the same richness. The evolution of the characteristic luminosity of the luminosity function can be described by a passively evolving population formed in a single burst (e.g. Lin et al. 2006). In this study, we derive $m^{\star}(z)$ from the passive evolution of a burst galaxy with a formation redshift $z_{\text {form }}=3$ taken from the PEGASE2 library (burst_s C86_zo. sed; Fioc \& Rocca-Volmerange 1997). It is calibrated using the value of $K^{\star}(z=0.25)$ derived by Lin et al. (2006) from an observed cluster sample. The choice of $\delta$ mag is critical as it sets the redshift limit $\left(z_{\text {lim }}\right)$ of the final cluster sample through the relation $\mathrm{m}^{\star}\left(z_{\text {cluster }}\right)+$ $\delta$ mag $=\operatorname{mag}_{\text {lim }}$, where $\operatorname{mag}_{\text {lim }}$ is the survey apparent magnitude limit.

\section{APPLICATION TO DES-Y 1 SURVEY}

\subsection{Running WaZP cluster finder}

As described in Section 2, the DES-Y1 survey is split into two regions ('SPT' and 'S82'), for which two galaxy VACs are produced to feed the WaZP pipeline. These catalogues are built based on the $i$-band, chosen here as a reference band both for star-galaxy separation, and for defining apparent magnitude cuts. Given this selection, cluster detection was performed with the same setting independently from the position on the sky, assuming a sufficient homogeneity over the whole survey. This is an approximation as we have seen above that in some regions magnitude completeness limit can be lower by as much as 1 mag.

The redshift limit of the constructed WaZP sample is constrained by the depth of the survey reference band. It also depends on the adopted definition of the richness estimate and in particular, the adopted magnitude limit used to count galaxies entering the richness. We assume here that the same cluster, seen at two different redshifts, would get the same richness by counting its galaxies down to an apparent magnitude $\mathrm{m}_{\mathrm{i}}{ }^{\star}(z)+$ $\delta_{\text {mag }}$, where $\delta_{\text {mag }}$ is a fixed quantity and $\mathrm{m}_{\mathrm{i}}{ }^{\star}(z)$ is defined in Section 3. This can be achieved as long as this quantity remains lower than the $m_{i}$-band depth of the survey. In the present case, richnesses are chosen to be computed including galaxies down to $\mathrm{m}_{\mathrm{i}}^{\star}+1.5$.

Based on the above considerations, given that some regions are not deeper than $m_{i}=22.27$, there is an upper redshift limit, $z=$ 0.60 , above which richnesses start to become incomplete depending on the survey location. At the limiting magnitude of the galaxy VAC, $m_{i}=23$, which corresponds to a redshift limit $z=0.76$, richnesses are complete within only 2 per cent of the survey area. Based on the $10 \sigma i$-band survey depth map (Section 2), we have derived a map indicating our local cluster $z_{\max }$ at each position of the survey, that is reported in the WaZP cluster catalogue. Detection is performed to slightly larger redshifts $(\sim 0.9)$, but for clusters that would be detected beyond their local $z_{\max }$, their galaxy luminosity function is not sampled homogeneously across redshifts and therefore richnesses for these clusters would require some correcting factor. 


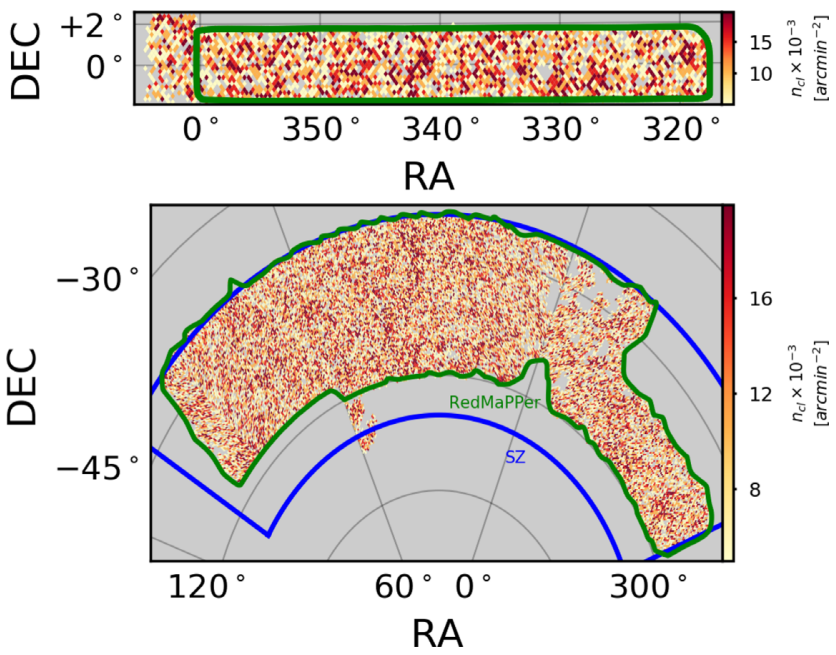

Figure 7. Projected density of WaZP clusters for both Y1-S82 (top) and Y1-SPT (bottom) regions of DES first year data release. The green line is the contour of the redMaPPer $\mathrm{Y} 1$ catalogue and the blue line is the region of detection for the SPT survey (Bleem et al. 2015).

In this paper, we are not introducing such a correction, and therefore richnesses are consistent over the whole survey only up to $z=0.60$. As a lower redshift limit for cluster detection, we adopted in this paper the value $z_{\min }=0.05$.

Besides the considerations on redshift limits above, we also need to assess the global quality of our photometric redshifts and determine the photometric redshift slicing strategy on running WaZP. As can be seen in the right-hand panel of Fig. 5, the average bias remains relatively modest and the scatter roughly constant in the adopted redshift range. These properties should not prevent cluster detection in general. Note, however, that this point will be discussed in more details in Section 6.

Operationally, the WaZP cluster finder runs on small sections of the sky. The LIneA Science Portal manages data tiling, launches the code on each tile on parallel cores, and concatenates the final catalogues, both clusters and galaxy members. The data tiling consists in dividing the survey in overlapping rectangular tiles of typical area $20 \mathrm{deg}^{2}$. The overlaps are set to assure a tiling independent cluster detection for clusters with redshifts larger than 0.05 that would fall at the intersection of two tiles.

\subsection{The WaZP cluster catalogue}

The WaZP pipeline was run on the DES Y1-S82 and Y1-SPT regions defined above. This results in the detection of cluster candidates, not confirmed galaxy clusters. However, these candidates will be referred to as 'WaZP clusters' throughout the paper for simplicity. For the combined sample, it led to the detection of 60547 clusters in the redshift range $z=0.05$ to $z=0.91$ with richness $N_{\text {gals }} \geq 5$, corresponding to densities of 40.47 and 39.45 clusters deg $^{-2}$ for Y1S82 and Y1-SPT, respectively. If we restrict to a sample with more reliable redshifts and complete richnesses i.e. $0.1 \leq z \leq 0.60$, we find 39439 clusters, with a higher consistency in cluster densities with

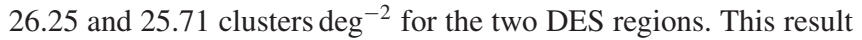
supports strongly the high homogeneity over the sky of the galaxy VAC construction, including photometric redshift computation, as well as the subsequent cluster detection. This can also be seen in Fig. 7 where the projected distribution of detected clusters on the sky is shown. A description of the WaZP cluster catalogue is provided on LIneA's website ${ }^{4}$.

The ranges of richnesses and redshifts covered by WaZP clusters are shown in Fig. 8. From the colour coded SNR, we can see that for a given richness, as expected, the SNR decreases with redshift. This is mainly due to the increasing scatter in the photometric redshifts leading to an increase of the mean background density of galaxies. We see that above redshift 0.60 , the number of rich clusters start to diminish rapidly, and above 0.76 there is only one cluster with richness greater than 100 .

The redshift distribution of WaZP clusters is shown in Fig. 9. The global bell shape of the counts looks as expected except for a sharp concentration of clusters at $z \sim 0.45$, similar to that observed on the galaxy photometric redshift distribution of Fig. 4. This peak becomes more prominent for lower richness systems. In the next sections, we investigate further the nature of this peak.

There are several ways to estimate the quality of the WaZP redshifts $\left(z_{\mathrm{W} a \mathrm{ZP}}\right)$. They can be compared to known cluster redshifts as shown in the next section, or, as done here, cluster members can be cross-matched with available spectroscopic galaxy samples. The adopted procedure here is to search for all galaxies with spectroscopic redshifts within $0.5 \mathrm{Mpc}$ around each detected cluster and likely to be cluster members. We considered that a cluster could be associated with a spectroscopic redshift if at least five galaxies were found within a range of $\pm 2000 \mathrm{~km} \mathrm{~s}^{-1}$. The selected velocity window is the one that maximizes the number of spectroscopic galaxies. The cluster spectroscopic redshift is then defined as the median of the redshifts in that window. We also associated a redshift in the case the central WaZP cluster galaxy has a spectroscopic redshift. This may lead to a few outliers but increases statistics by a factor of 10 . Based on public spectroscopic surveys, $131 \mathrm{WaZP}$ clusters covering the redshift range $z=0.05$ to $z=0.9$ could be associated to a spectroscopic redshift with at least 5 concordant redshifts, and 1859 clusters could be associated to a spectroscopic redshift based on their central galaxy. In Fig. 10, the comparison with WaZP redshifts is shown. Both spectroscopic redshift assignments led to the same statistical differences with WaZP redshifts: an average bias of $\sim 0.014$ and scatter of $\sim 0.026$.

Fig. 11 shows the evolution of the volume density of clusters with redshift, with Poisson error bars. We can see that the density for both Y1 regions agree with each other for both richness cuts.

\section{COMPARISON TO OTHER CLUSTER CATALOGUES}

In this section, we compare the WaZP Y1A1 clusters identified in the previous section to those derived by other methods covering the same region. A first comparison is made with clusters detected by the South Pole Telescope (SPT) survey via the Sunyaev-Zel'dovich (SZ) effect (Bleem et al. 2015). A second comparison is done with clusters detected by the redMaPPer optical cluster finder, based on the same photometric data but using an algorithm searching for overdensities of red sequence galaxies (Rykoff et al. 2014). These comparisons are based on matching clusters from two different catalogues. This is a complex operation that has led to a variety of proposed algorithms (e.g. Gerke et al. 2005; Knobel et al. 2009; Cucciati et al. 2010; Gerke et al. 2012; Euclid Collaboration 2019). We point out that adopting one algorithm or another or using different configurations of the same may result in different cluster associations.

\footnotetext{
${ }^{4}$ https://www.linea.gov.br/catalogs/wazp/
} 


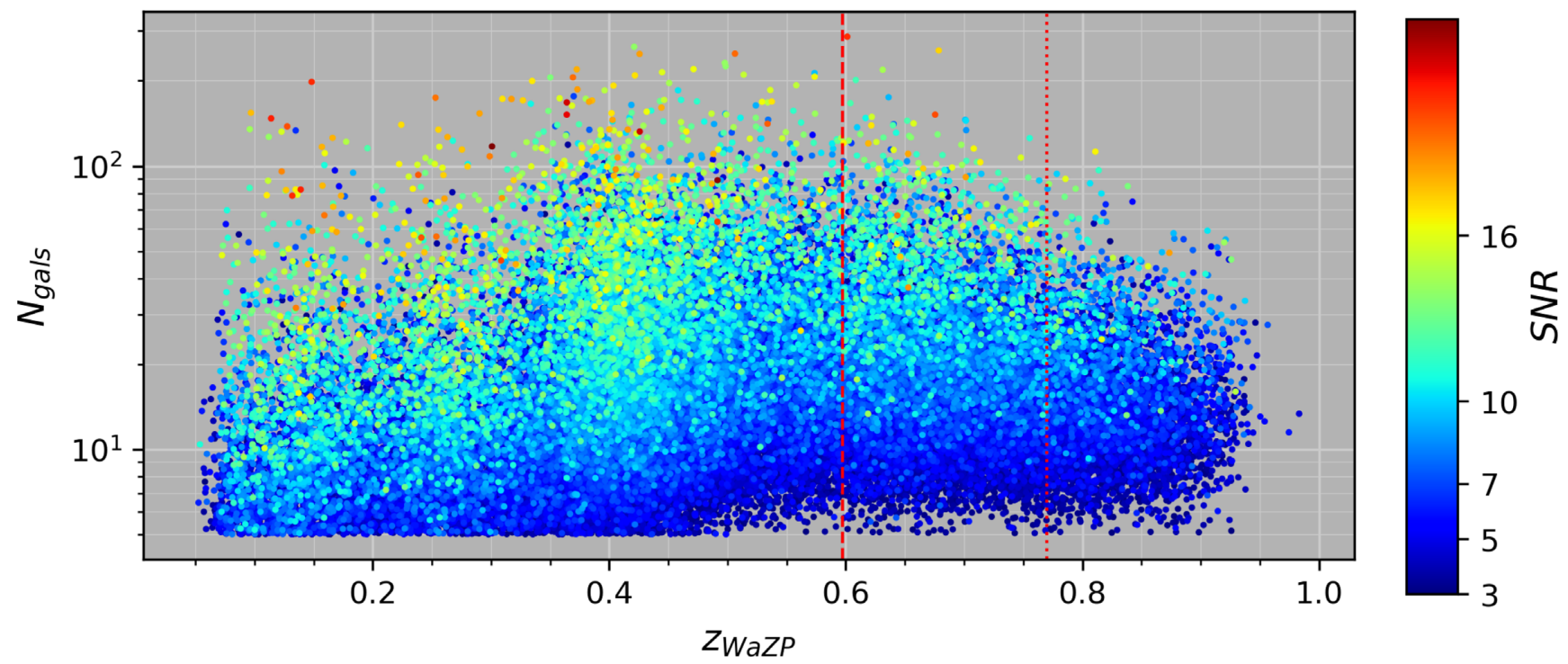

Figure 8. Richness as a function of redshift for WaZP clusters colour coded by signal-to-noise ratio. The dashed line indicates the redshift (0.60) above which cluster richnesses start to become incomplete and the dotted line marks the redshift (0.76) above which all clusters have incomplete richnesses.

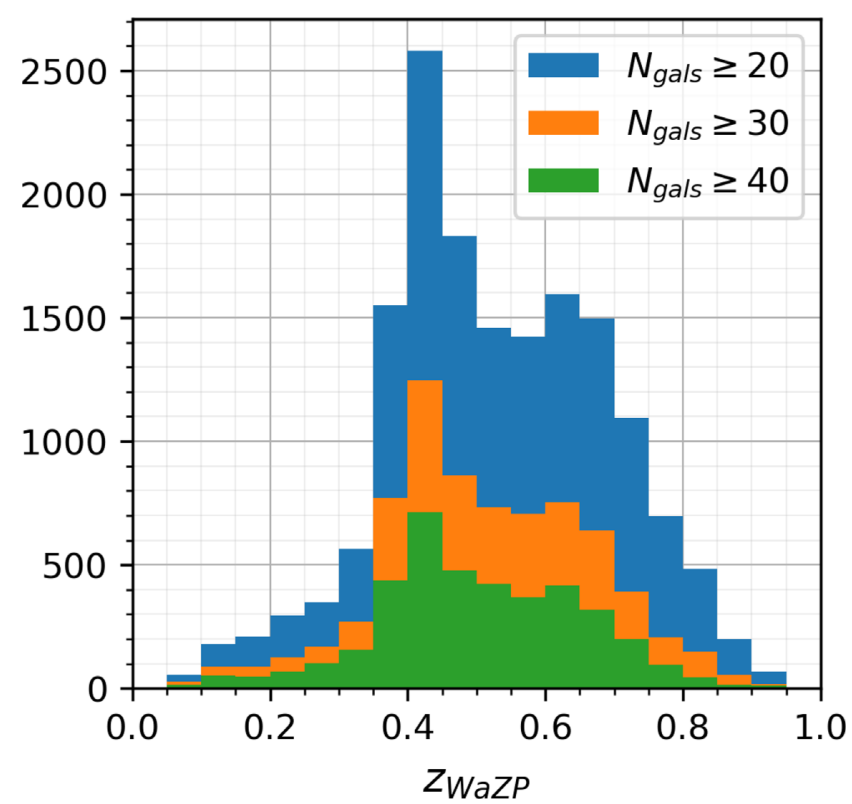

Figure 9. The counts distribution of WaZP clusters detected in the combined Y1-S82 and Y1-SPT DES regions as a function of cluster redshift for three different richness cuts.

In particular, when a given cluster can potentially be connected to several counterparts. As detailed below, we have experimented with several of these approaches and finally adopted a hybrid iterative procedure to optimally solve multiple matches. This appeared to be the only way to avoid having systems left unmatched due to a wrong matching of their obvious counterpart. Several such cases appeared in particular with interacting clusters for which richness rankings were reversed. The resulting pairing seems, from intensive visual inspection, optimal for addressing statistically the different properties of the commonly detected clusters (centring, redshift), and evaluating systems without any counterparts. In particular, our matching procedure allowed us to decrease the number of incorrect matches of rich clusters on each side significantly.

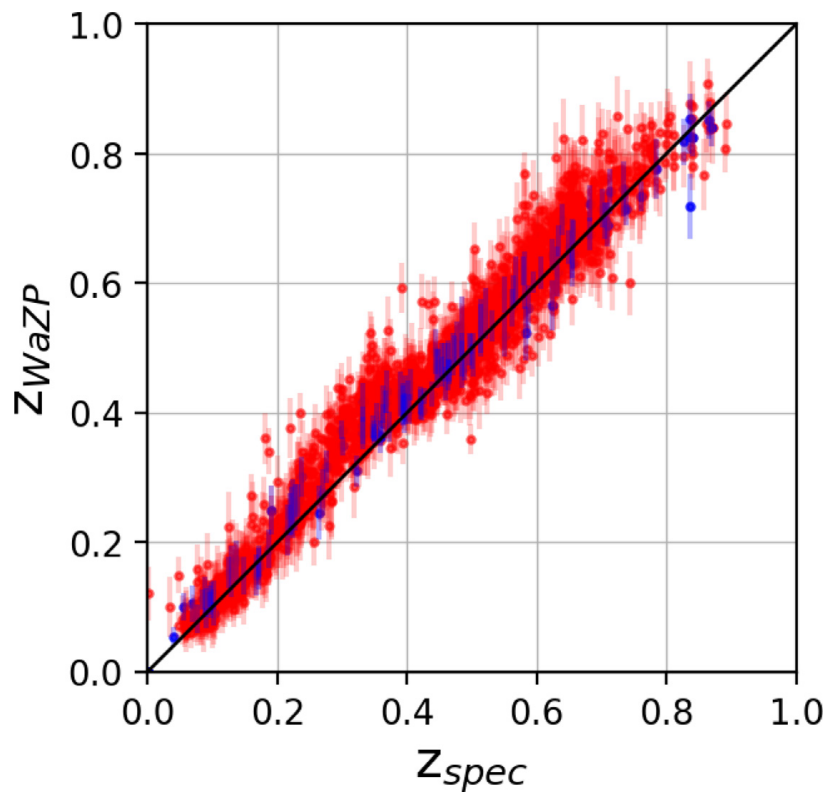

Figure 10. Comparison of cluster redshifts as derived by WaZP with estimated spectroscopic redshifts. WaZP cluster members are cross matched to all publicly available spectroscopic redshifts falling in the DES footprint. A cluster spectroscopic redshift is derived each time at least 5 concordant redshifts (within $\pm 2000 \mathrm{~km} \mathrm{~s}^{-1}$ ) are found within $0.5 \mathrm{Mpc}$ to the cluster centre (blue points, 131 clusters), or if the WaZP central galaxy has a spectroscopic redshift (red points, 1859 clusters).

In this paper, we use a cylindrical matching where we require the angular distance of cluster centres to be smaller than some defined length (be it their respective radii or a fixed physical distance), and their redshift separation to be constrained by the typical redshift errors from both samples. In carrying out this comparison, some issues have to be considered. First, the cluster radius definition for each cluster sample may be different. Secondly, we must define the redshift window to be used. It should be large enough to take into consideration the errors in photometric redshift assigned to clusters 


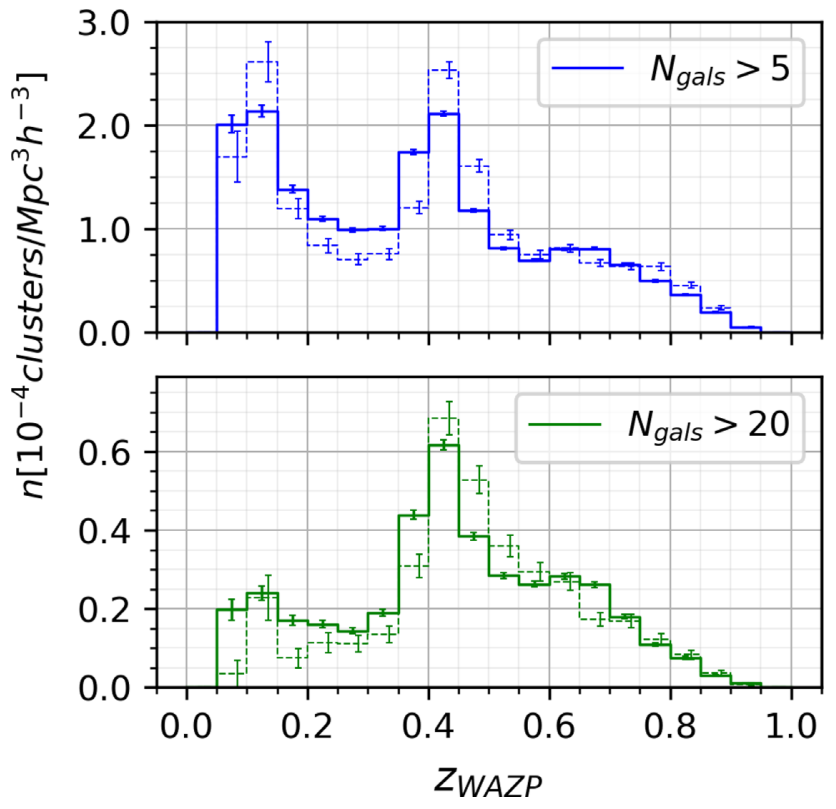

Figure 11. Volume density of WaZP clusters as a function of redshift in Y1-SPT (solid line) and Y1-S82 (dashed line) regions with different richness thresholds. The error bars correspond to Poisson noise.

in both samples. However, a large window may lead to an increased number of multiple matches that need to be resolved as discussed in more detail below. Finally, it is crucial to ensure that the projected area of overlap of the samples is properly taken into account. To do that, footprints of the samples are used to flag clusters falling outside the overlapping regions or near their edges. This flag is useful when unmatched clusters are near the edges, in which case they can be removed from the matching statistics.

As mentioned above, we compare the combined Y1-S82 and Y1SPT clusters identified by WaZP with those in the SZ and redMaPPer samples. In the first case, we take the SZ sample as reference, treating the SZ clusters as true representatives of the underlying mass distribution and test how well these systems are recovered. In the redMaPPer case, we investigate the unmatched cases in both directions to understand the specificities or the possible limitations of each algorithm.

\subsection{WaZP versus SPT clusters}

The SZ sample (Bleem et al. 2015) covers an area of $2500 \mathrm{deg}^{2}$ (seen in bottom panel if Fig. 9), within which 516 clusters (out of 677 candidates) were detected with signal-to-noise above 4.5. In Bleem et al. (2015), it is stated that the catalogue is highly complete for $M_{500^{c}} \geq 7 \times 10^{14} M_{\odot} h^{-} 1$ and $z \geq 0.25$. It is also mentioned that there were a number of optical followups to confirm these clusters. Therefore, this catalogue will be utilized to validate the detectability of WaZP regarding massive clusters. We only use the $331 \mathrm{SZ}$ clusters that have information on mass and redshift, and are located within the overlap with DES (external envelope of the DES Y1-SPT region). We should stress that the redshifts assigned to SZ clusters from Bocquet et al. (2019) are both spectroscopic (106) and photometric (225).

We considered a one-way match, taking SZ clusters as reference and we looked for WaZP clusters falling within SPT clusters radii. The adopted radius is $R_{200}$, the radius where the average cluster overdensity is 200 times the critical density, i.e. $\Delta=$ $3 M_{200} / 4 \pi R_{200}^{3} \bar{\rho}_{\text {crit }}(z)=200$. It was computed from the available

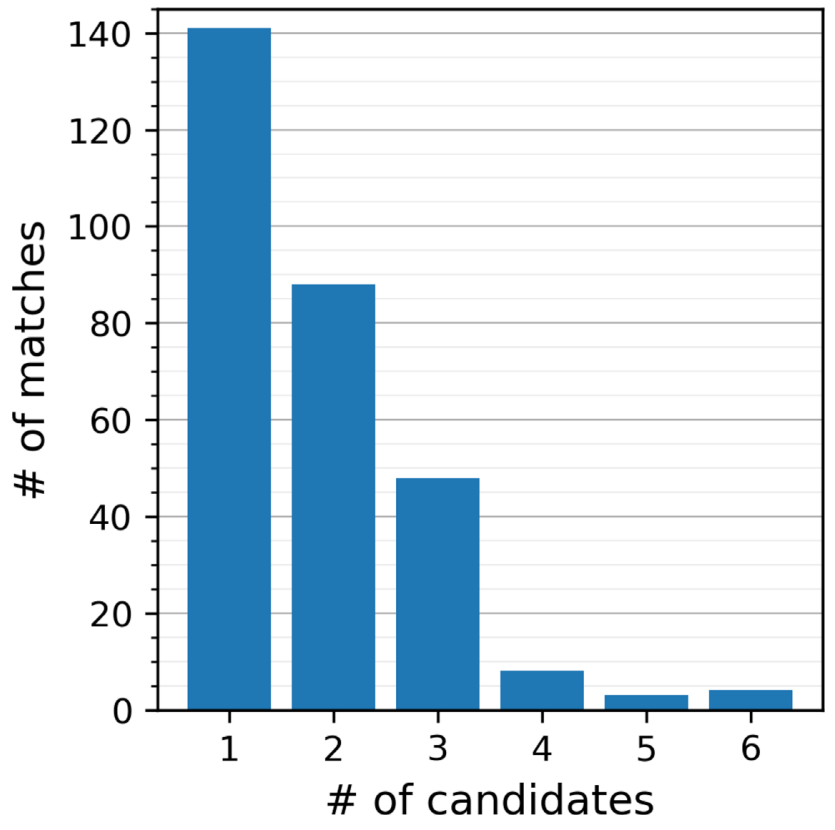

Figure 12. Number of WaZP clusters found to match individual SZ clusters.

values of $M_{200}$ and converted to an angular radius $\theta\left(R_{200}\right)$ using Planck Collaboration XIII (2016) cosmology. Here, $\theta_{200}=$ $R_{200} / D_{A}(z)$, where $D_{A}(z)$ is the comoving angular diameter distance to the cluster redshift $z$. For a match to happen, the redshift separation also has to fall within the interval defined by the combined redshift errors. This interval was defined as $3 \sigma$, where $\sigma$ is the sum of redshift errors provided by the two catalogues. The resulting matching cylinder is quite large in order to account for possible centring offsets between the two wavelength domains and large redshift discrepancies. The statistical comparison, a posteriori, of the differences in centring and redshifts of the matched systems, allows us to evaluate the adopted matching criteria.

Applying this method to the SZ and WaZP samples, we find that 292 SZ clusters (out of 331) have at least one WaZP counterpart. Among these, 141 have only one candidate for matching, while the rest have a multiplicity function as shown in Fig. 12. The large fraction of multiple matches is not surprising considering the very different selection functions of the two samples, their relative densities, and the adopted matching criteria. The multiple WaZP matches were resolved by choosing the richest associated counterpart.

Out of the 39 unmatched SZ clusters, 12 are located near the WaZP footprint edges and 27 have redshifts beyond $z=0.76$, where WaZP cluster finder reaches its expected limit of completeness for DES-Y1 ( 9 of those have $z>1.1$, and are completely beyond WaZP reach). There was one unmatched SZ cluster (SPT-CLJ22185532) with $z=0.77$, just above the redshift limit for WaZP clusters with complete richnesses. However, the local $z_{\max }$ at this cluster position is 0.71 and, upon visual inspection, we found no clear visible optical counterpart.

In Fig. 13, we show the characteristics of the matching for three mass bins, both in terms of angular separation (left-hand panel) and redshift separation (right-hand panel). The average distance of WaZP-SZ centres is $\langle\Delta \theta\rangle=0.16 R_{200}$, with 80 per cent of clusters within $0.2 R_{200}$ and 95 per cent within $0.8 R_{200}$. If we consider the different mass bins in the figure, there is a small systematic improvement on $\langle\Delta \theta\rangle$ for higher masses $(0.177,0.178$, and 0.134 , respectively), even though there is only $\approx 100$ clusters per mass bin. 

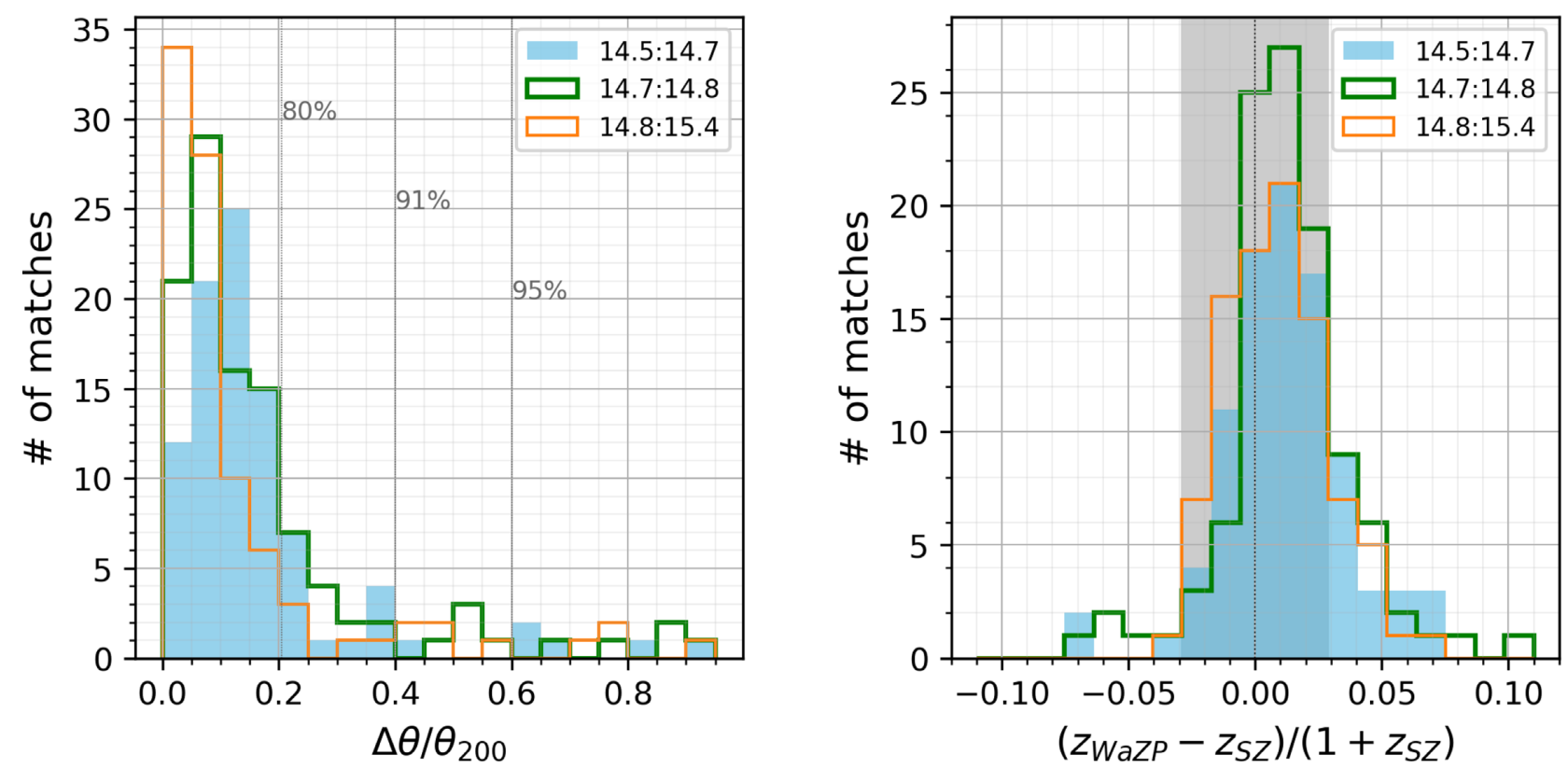

Figure 13. Distribution of angular distances (left-hand panel) and redshift separations (right-hand panel) of WaZP-SZ matched clusters binned by $\log \left(M_{200}\left[M_{\odot} h^{-1}\right]\right)$. The grey shaded region on the right-hand plot is the combined average photometric redshifts uncertainties of the matched clusters from both catalogues divided by $1+z_{\mathrm{SZ}}$.

We also see that there is a reasonable agreement in redshift, with 79 per cent of matches within the average redshift uncertainties of the clusters $\left\langle z^{\mathrm{err}}\right\rangle=\sqrt{\left\langle z_{\mathrm{W} a \mathrm{ZP}}^{\mathrm{err}}\right\rangle^{2}+\left\langle z_{S Z}^{\mathrm{err}}\right\rangle^{2}}$ (grey shaded region). There is also a slight improvement on redshift scatter and bias was we look at higher mass bins.

We also compare the photometric estimates of WaZP redshifts with those assigned to SZ clusters. As 93 of the matched SZ clusters have been assigned a spectroscopic redshift (Bocquet et al. 2019), we can assess the accuracy of the estimated WaZP redshifts. Fig. 14 shows the distribution of redshift separations (left-hand panel) and relation of redshifts (right-hand panel), splitting the SZ cluster redshifts into spectroscopic and photometric subsamples. As can be seen, WaZP redshifts show a good agreement with SZ spectroscopic redshifts and some residual relative bias when compared with SZ photometric redshifts. A quantitative description is provided in Table 2, which gives in column (1) the type of sample; in column (2) the number of clusters and in columns (3) and (4) the bias and the scatter, defined as the mean and standard deviation of $\left(z_{\mathrm{W} a \mathrm{ZP}}-z_{\mathrm{SZ}}\right) /\left(1+z_{\mathrm{SZ}}\right)$, and column (5) is the combined redshift errors $\left\langle z^{\mathrm{err}}\right\rangle /\left(1+z_{\mathrm{SZ}}\right)$. When compared to photometric redshifts, we measure a relative bias of $\sim 0.015$ and a scatter $\sim 0.029$, a value similar to the combined redshift error 0.030. However, when comparing to SZ clusters with spectroscopic redshifts, WaZP redshifts are almost unbiased (bias $=0.007)$, and show a significantly lower scatter $(\sigma=0.017$, also compatible with the combined errors 0.015 ), corresponding to roughly half the average galaxy photometric redshift scatter. It is interesting to note that in all samples, the scatter was very close to the redshifts uncertainties, even though the matching conditions only imposed a redshift difference of three times the errors. From the right-hand panel of Fig. 14, it can also be seen that the moderate average bias is actually mainly due to low redshift clusters $(z \lesssim 0.4)$.

\subsection{WaZP versus redMaPPer}

In this paper, we also match WaZP clusters with those detected by the redMaPPer algorithm. Here we use the redMaPPer volume limited cluster catalogue of DES-Y1 presented in McClintock, T. \& Varga, T. N. et al. (2019) which consists of 83238 clusters with richness $\lambda \geq 5$ found in the Y1-SPT and Y1-S82 regions (seen in Fig. 9) over the redshift range 0.1-0.95. This volume limited catalogue considers clusters for which the local $z$-band depth assures a complete galaxy catalogue down to the adopted magnitude limit of the richness definition. The variable $z$-band depth translates into a variable redshift limit $\left(z_{\max }\right)$ map that characterizes the cluster sample. When evaluating the recovery rates of clusters at high redshifts, we also consider the full redMaPPer cluster catalogue over the same region, defined by a constant $z_{\max }=0.95$ and a richness threshold of 20 .

\subsubsection{Differences in the detection algorithms}

redMaPPer is an optical cluster finder based on the detection of spatial overdensities of red sequence galaxies (Rykoff et al. 2016). Although WaZP does not make any assumption relative to the cluster galaxy population when searching for galaxy overdensities, we do expect these two algorithms to yield similar samples up to redshifts $\sim 0.7$, at least when considering the richest systems. However, a number of differences can be expected in the cluster characterization for several reasons. First, as it was stressed above, galaxies used for searching for overdensities are not selected in the same way. redMaPPer selects them based on colours whereas WaZP selects them based on redshifts. Secondly, the two algorithms differ in defining cluster centres. In the case of REDMAPPER centres are associated to a bright galaxy with some probability of being a central galaxy, whereas WaZP defines the centre as a centroid. Note however, that, as described above, WaZP moves the centre to the brightest cluster member position if its distance is less than $100 \mathrm{kpc}$, which happens here for 68 per cent of the WaZP clusters. Thirdly, REDMAPPER redshifts are assigned based on an empirical modelling of red sequence colours, whereas WaZP assigns redshifts based on a concentration in photometric redshift space including all galaxy types at the cluster location. Finally, we also expect differences on 

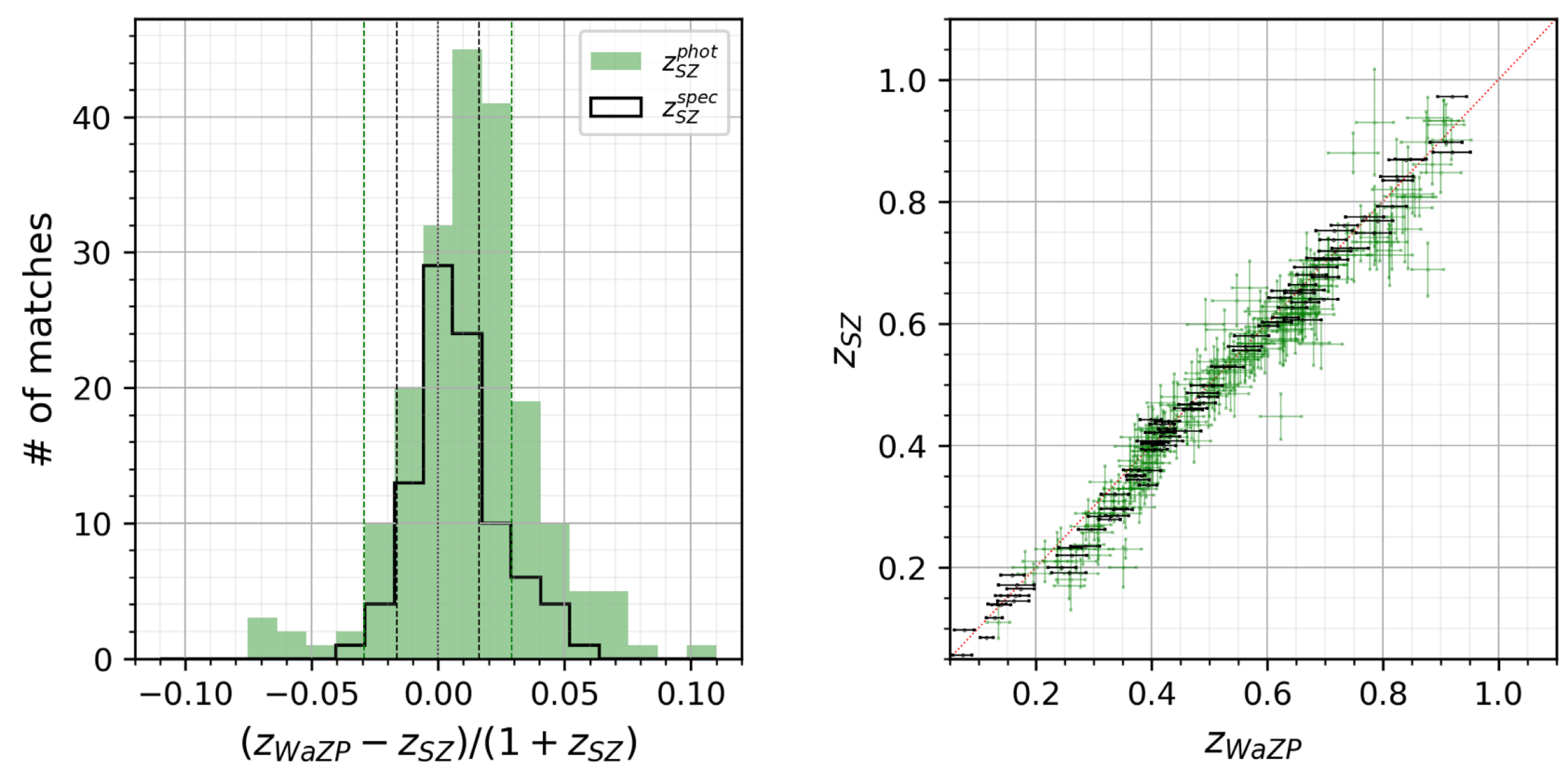

Figure 14. Distribution of redshift separations (left-hand panel) and relation between the redshifts (right-hand panel) of WaZP-SZ matched clusters split into spectroscopic and photometric redshift samples.

Table 2. Bias, scatter, and combined redshift errors of WaZP$\mathrm{SZ}$ matched clusters for different subsamples as described in the text.

\begin{tabular}{cccccc}
\hline & Sample & $N$ & Bias & Scatter & Errors \\
\hline & All redshifts & 292 & 0.012 & 0.026 & 0.025 \\
{$[\mathrm{~h}]$} & Phot z only & 200 & 0.015 & 0.029 & 0.030 \\
& Spec z only & 92 & 0.007 & 0.017 & 0.015 \\
\hline
\end{tabular}

how each cluster finder performs in terms of deblending, or in terms of fragmentation and overmerging.

The above effects make the matching between the two samples non-trivial, since the key elements to perform a proximity matching, like centring and redshift, can have distinct behaviour, that may not lead to a unique solution. Despite its complexity, it should be able to provide us with a measure of the statistical consistency of the two catalogues. It should also help us infer a lower limit for centring uncertainty, as both cluster finders have optical centring estimations. Finally, by carefully dealing with footprint coverage and edge effects, it should allow us to identify a number of missing systems and provide feedback on the respective selection functions, on possible ways to improve cluster detection algorithms and improve aspects of the construction of the underlying galaxy catalogue.

\subsubsection{Matching procedure}

In contrast to what was done in the comparison between WaZP and SPT clusters, here, each cluster sample is considered as a reference to the other. Therefore, not only we consider a one-way match using redMaPPer as a reference (redMaPPer-matched), but we also analyse the case where WaZP (WaZP-matched) is the reference catalogue. These one-way matches are used to investigate the fraction of missed detections. In addition to the one-way matches, in order to estimate differences in cluster properties (e.g. centring, redshift, richnesses), we also carry out a two-way (unique) match, for which it is required that both one-way matches point to the same cluster.
We recall that the cluster matching is performed within a specified redshift window. Following what is done in Section 5.1 when matching with SZ clusters, this window was first defined considering the sum of the redshift errors provided for each cluster in the different samples. However, while visually inspecting a sample of unmatched systems, it was noticed that for relatively low redshifts $(z \lesssim 0.4)$, obvious pairs (i.e. sharing exactly the same centre without any other overdensity on the line of sight) were not associated due to large redshift discrepancies. This fact is not surprising due to the systematic errors in photometric redshifts occurring at low redshifts. In order to take this effect into account, we used an empirical approach to define the redshift window for matching. We first matched systems by angular centre proximity only, without a redshift window, but imposing the centre angular positions to be closer than $0.05 \mathrm{Mpc}$, computed at the largest redshift of the cluster pair. The clusters were ranked by richness, and when multiple candidates were found (about 10 per cent of the time for WaZP clusters and 24 per cent for redMaPPer), the richest candidate was selected. As judged by eye inspection, with this criterion, most matches refer to the same system.

Fig. 15 presents the resulting relation between redshifts for WaZP and redMaPPer matched clusters. As can be seen, this relation shows some deviations from a linear relation, in particular at WaZP redshift $\sim 0.4$, where redshifts are distributed from 0.2 to 0.5 , so a large scatter. This issue is discussed in more details in the following section. Based on this plot, we defined a new redshift window to carry out the matching, which corresponds to the union of the 99 percentile of the redshift differences using redMaPPer as reference (as it is covering a smaller redshift baseline) and a $3 \sigma(1+z)$ scatter.

Matching the catalogues by considering the resulting large redshift window combined to larger angular radii than in Fig. 15 unavoidably leads to a large fraction of multiple associations. Resolving these multiples by selecting the richest available system on both sides resulted in many false matches. An emblematic case that appeared several times in our visual inspections, is the case of interacting clusters of similar richnesses. Both cluster finders would detect the two components but not necessarily with the same richness 


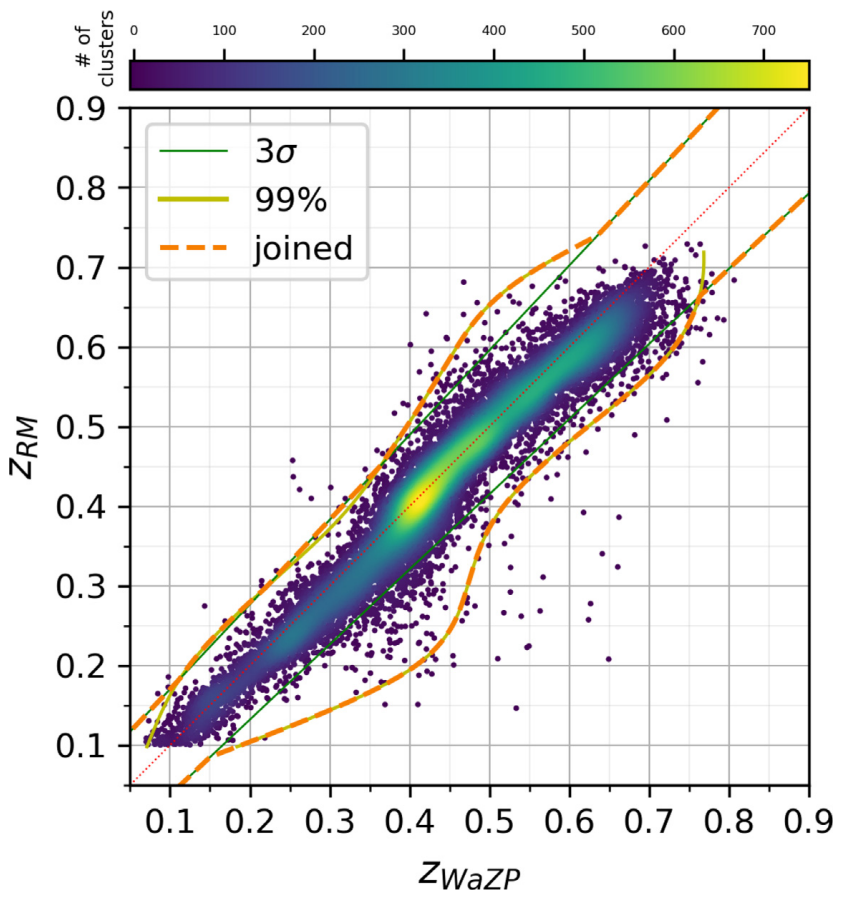

Figure 15. Redshift relation for WaZP-redMaPPer matched clusters within $50 \mathrm{kpc}$. The yellow lines are the 99 per cent percentiles, the green lines are the $3 \sigma$ levels, and the orange dashed lines are the union of the two, which will be used for the last step of matching.

ranking. In that case the matching could lead to one mismatch and one unmatched cluster, or more mismatches due to a cascade effect. However, in the case of absence of an interacting system or if neighbouring systems are much poorer, the richness ranking is more adequate.

We found that for maximizing the number of correct associations, the best option is to go beyond a single matching rule. Therefore, we decided to perform the matching following a several steps process where the most unambiguous pairs are matched first and then proceed to the rest of the list. The steps are detailed below.

It was determined empirically that a four-step process is optimal, where, at each step, the matching would only be performed on clusters not previously matched. In the first step, we do not consider the clusters' redshift, and match all clusters that have the exact same centring (which happens when the two cluster finders are centred on the same galaxy). By construction, each one way match finds the same corresponding pairs, therefore all cluster pairs found here will also be a match in the two way matching. This led to a total of 15534 matched clusters. In the second step, remaining clusters are matched within an angular distance of $300 \mathrm{kpc}$ (computed at the lowest redshift of the pair) from each other, and a redshift difference less than $1 \sigma_{z}$ (computed from Fig. 15). When more than one candidate is found, the richest one is considered to be the correct match. This step also results in having the same number of matched clusters for both one way matches, adding an extra 6431 matched clusters in each catalogue. The third step expands on the second one with a $3 \sigma_{z}$ window, leading to additional 3783 matches in each catalogue. In the last step, we match the remaining clusters with the empirical redshift window shown in Fig. 15 and use the radius provided by each cluster finder as a parameter for angular distance. Here, the matching is not symmetric and results in another 4915 and 3027 matched clusters for redMaPPer and WaZP, respectively. Hence, we obtained a total of 30663 one-way matches for redMaPPer and and
28775 for WaZP. We note that, in this four-step matching, if we do not remove matched clusters at each step and allow for multiple matches, we obtain 32467 redMaPPer matched clusters and 33498 WaZP matched clusters.

Finally, two-way matches are obtained when the two one-way matches point to each other. This results in 28621 redMaPPer-WaZP cross-matched clusters.

\subsubsection{Comparison of the matched clusters}

We start by comparing the individual properties of the matched clusters (i. e. centring, redshift, and richness). Cross-matched clusters (28 621 pairs) will be used in this evaluation, as a reliable one-toone correspondence between clusters is required. Fig. 16 shows the distribution of angular separation (left-hand panel) and the redshift difference (right-hand panel) of two-way matched clusters. As it can be seen, all matched clusters are well within the mean radius of the clusters $(\sim 600 \mathrm{kpc})$, that was used in the last step of the matching. In 56 per cent of the cases, clusters have the exact same centre. Those occur when WaZP defines the same BCG as redMaPPer to be its centre. The average distance of central position for the clusters that do not share the same centre is of $86 \mathrm{kpc}$, with 86 per cent of matched clusters within $100 \mathrm{kpc}$ of each other and over 99 per cent within $300 \mathrm{kpc}$. The angular separation only shows a very weak tail beyond the typical cluster core radius. In addition, we note that the centring statistics do not seem to depend significantly on the richness.

Turning to the distribution of the redshift separations, we find that for the vast majority of pairs ( $>99$ per cent), the redshift separation is well within one third of the redshift window used in the last step of the matching, which has an average size of $\sim \pm 0.15(1+$ z). Additionally, we find over 75 percent of pairs with redshift separation within the combined uncertainty of the cluster redshifts $\left\langle z^{\mathrm{err}}\right\rangle=\sqrt{\left\langle z_{W a Z P}^{\mathrm{err}}\right\rangle^{2}+\left\langle z_{\mathrm{RM}}^{\mathrm{err}}\right\rangle^{2}}=0.028$ (grey shaded region). We note, however, a small average redshift bias of 0.010 (Table 3) exists between WaZP and redMaPPer, with redshifts derived by WaZP being on average slightly larger than redMaPPer redshifts. In Fig. 17, redMaPPer and WaZP redshifts are compared, showing that the bias is mainly due to a significant fraction of $z \sim 0.3$ clusters that were pushed to $z_{\text {wazp }} \sim 0.4$, an effect that is discussed in the next section. Additionally, we see on the right-hand panel that this effect occurs mainly on poor $(\lambda<5)$ clusters. Overall, these results show that most matches are well within the ranges adopted in the matching procedure. It strongly supports the idea that we are detecting on average the same systems. We also note that the scatter is very similar to the combined redshift uncertainties in all richness limited samples (Table 3), and the biases are well within these values.

We now compare the values of richnesses as derived from the two algorithms. We do not expect them to be equal in average as they are derived with different definitions. redMaPPer richness $(\lambda)$ considers red sequence galaxies down to $0.2 \mathrm{~L}^{*}$ in the $z$-band, whereas WaZP richness $\left(N_{\text {gals }}\right)$ considers all galaxies down to $0.25 L^{*}$ in the $i$-band. Moreover these quantities are not necessarily computed in the same angular radius. Despite these differences, we expect some correlation between these two richness estimates. To perform this comparison, we restrict to two-way matched clusters with same centres and redshift offsets $\leq 0.02(1+z)$. We also considered clusters in the redshift range $0.1-0.6$ in order to assure richnesses to be complete for both cluster finders. Fig. 18 shows a strong correlation between the richnesses of the two cluster finders, with 

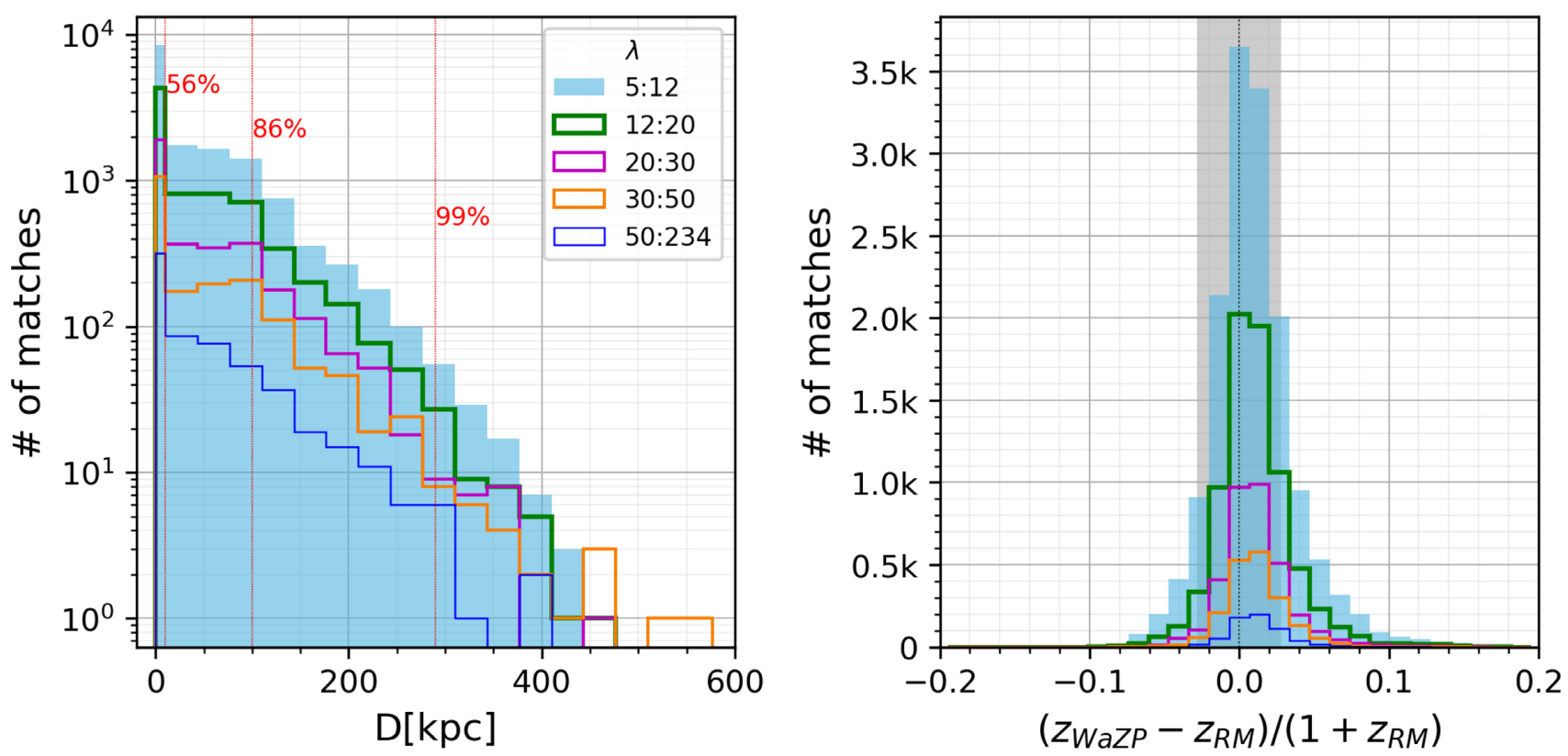

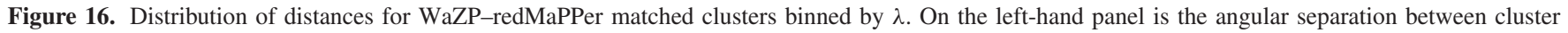

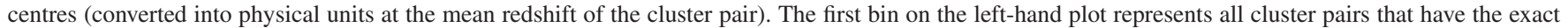
same position. On the right-hand panel is redshift differences and the grey shaded region is the average combined redshift uncertainties divided by $1+z_{\mathrm{RM}}$.

Table 3. Bias, scatter, and uncertainty of WaZP-redMaPPer matched clusters.

\begin{tabular}{lcccc}
\hline \multicolumn{1}{l}{ bin } & \# clusters & $\frac{z_{\mathrm{W} a \mathrm{ZP}-z_{\mathrm{RM}}}}{1+z_{\mathrm{RM}}}$ & $\frac{\sigma_{z}}{1+z_{\mathrm{RM}}}$ & $\frac{\sqrt{\left(z_{\mathrm{RM}}^{\text {err }}\right)^{2}+\left(z_{\mathrm{W} a \mathrm{ZP}}^{\text {err }}\right)^{2}}}{1+z_{\mathrm{RM}}}$ \\
\hline All & 28621 & 0.010 & 0.027 & 0.028 \\
$5-12$ & 15099 & 0.010 & 0.029 & 0.031 \\
$12-20$ & 7531 & 0.010 & 0.025 & 0.027 \\
$20-30$ & 3440 & 0.011 & 0.023 & 0.024 \\
$30-50$ & 1918 & 0.011 & 0.021 & 0.021 \\
$50-234$ & 632 & 0.014 & 0.019 & 0.018 \\
\hline
\end{tabular}

WaZP richnesses on average systematically larger than redMaPPer ones. To quantify the effect, we performed a linear fit in log space. To do so, cluster richnesses were first sliced in $\lambda$ and for each slice the mode of the smoothed distribution of $N_{\text {gals }}$ computed. This procedure minimizes the effect from Malmquist bias when constraining the relation between richnesses. The fit of the resulting $\left(N_{\text {gals }}, \lambda\right)$ pairs led to the relation:

$\log \lambda=(0.92 \pm 0.09) \log N_{\text {gals }}+(0.01 \pm 0.11)$.

An independent comparison of the two estimated richnesses is based on the cluster density given a richness threshold. This is shown as the blue dashed line in Fig. 18. Each point of this line provides the threshold in $N_{\text {gals }}$ and in $\lambda$ richnesses to obtain the same density in the two cluster samples, and is independent of any matching. It is remarkable that this measurement is very close to the mean relation between the two richness estimators. Another way to look at this is to compare directly the densities of the two cluster samples. In Fig. 19, we compare WaZP and redMaPPer cluster densities considering clusters with redshifts in the range 0.1-0.6 and with richnesses above a given threshold, where the threshold in $N_{\text {gals }}$ and $\lambda$ are related following equation (1) (e.g. $\lambda \geq 20$ is equivalent to $N_{\text {gals }} \geq 25$ ). Cluster densities are very similar over a very wide range of richnesses. This result supports the idea that, on average, the ranking of the two cluster samples by their richness is similar.

\subsubsection{Statistics of unmatched clusters}

We now evaluate the recovery rates between catalogues. Our main goal in this section is to check if each cluster finder could have missed a detection, therefore we take a very conservative approach to label clusters as unmatched. In principle, these rates could be computed considering a two-way matching. However, in that case, if a cluster of the first sample appears to be fragmented in the second one, the extra cluster will be counted as not recovered. Here, we wish to separate the absence of a counterpart from fragmentation, which should be treated separately. Therefore, we defined the recovery rate as the fraction of clusters having one or more counterparts in the matched cluster sample. Obviously, the absence of a match does not exclude completely the existence of a counterpart. Some systems could suffer from a strong miscentring, larger than tolerated by the matching criteria. Other systems could also suffer from edge effects that may occur at the periphery of the survey or close to a masked region within the survey. To minimize the latter, we do not consider unmatched clusters outside the intersection of the two cluster sample footprints (constructed as NSIDE $=4096$ Healpix maps) or unmatched cluster located in edge pixels. In addition, as redMaPPer removes clusters with over 20 per cent of their area masked, an equivalent consideration had to be made when looking for WaZP counter parts. Hence, unmatched clusters whose cover fraction was less than 80 per cent on the other catalogue's detection fraction footprint were also discarded from the analysis. These cover fractions were computed using the same weighted methodology as (Rykoff et al. 2014) considering the other catalogue footprint. Although this is not a major contribution for the values of the recovery fraction of WaZP clusters, ignoring this effect leads to lower recovery close to the footprint edges and holes. It is also important to note that, because both catalogues have a footprint with a variation on $z_{\max }$ at different locations, the computation of whether the cluster is inside the footprint or in a edge pixel and its cover fraction depends on the cluster position and redshift.

These cuts, based on the footprints and cover fraction, removed 16283 and 18737 redMaPPer and WaZP clusters without 

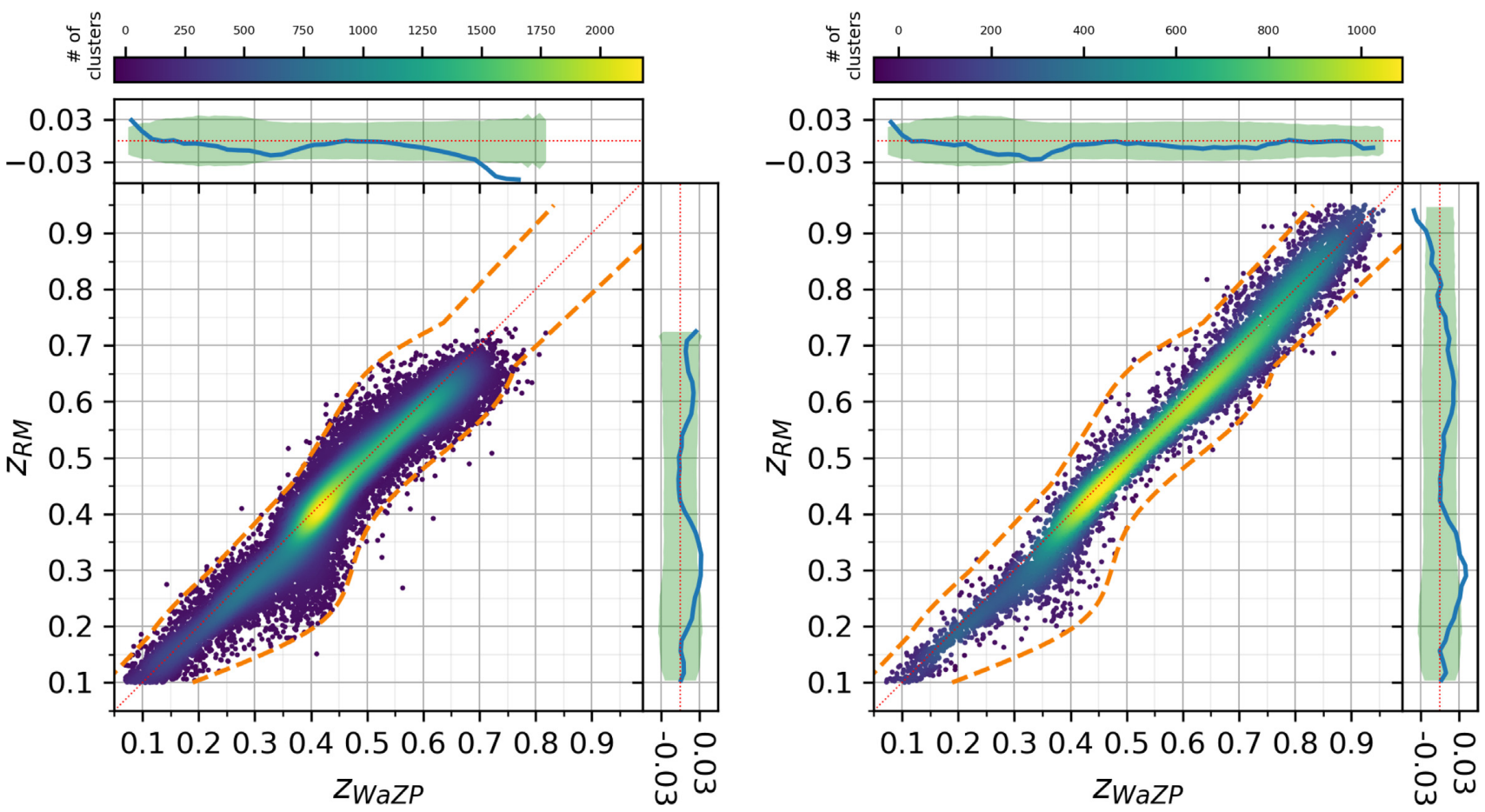

Figure 17. Redshift comparison of WaZP and redMaPPer matched clusters by the four-step match, with the colours corresponding to a count map. The left-hand panel is the matching to the redMaPPer volume-limited catalogue with $\lambda \geq 7$. The right-hand panel is the matching to the redMaPPer full catalogue $\lambda \geq 20$, showing many additional matches at redshifts above $\sim 0.7$. The matching of both catalogues was done as described in Section 5.2.2, with the yellow dashed lines being the windows defined in Fig. 15. The top and right blue lines are the bias of the redshift relations defined by $\left(z_{\mathrm{RM}}-z_{\mathrm{WaZP}}\right) /\left(1+z_{\mathrm{WaZP}}\right)$ and $\left(z_{\mathrm{WaZP}}-z_{\mathrm{RM}}\right) /(1$ $\left.+z_{\mathrm{RM}}\right)$, respectively. The green shaded regions in those panels are the average combined uncertainties the clusters redshifts $\left\langle z^{\mathrm{err}}\right\rangle=\sqrt{\left\langle z_{W a Z P}^{\mathrm{err}}\right\rangle^{2}+\left\langle z_{\mathrm{RM}}^{\mathrm{err}}\right\rangle^{2}}$. Comparing both panels, we can see that most outliers present at $z$ WaZP $\sim 0.4$ are low richness clusters.

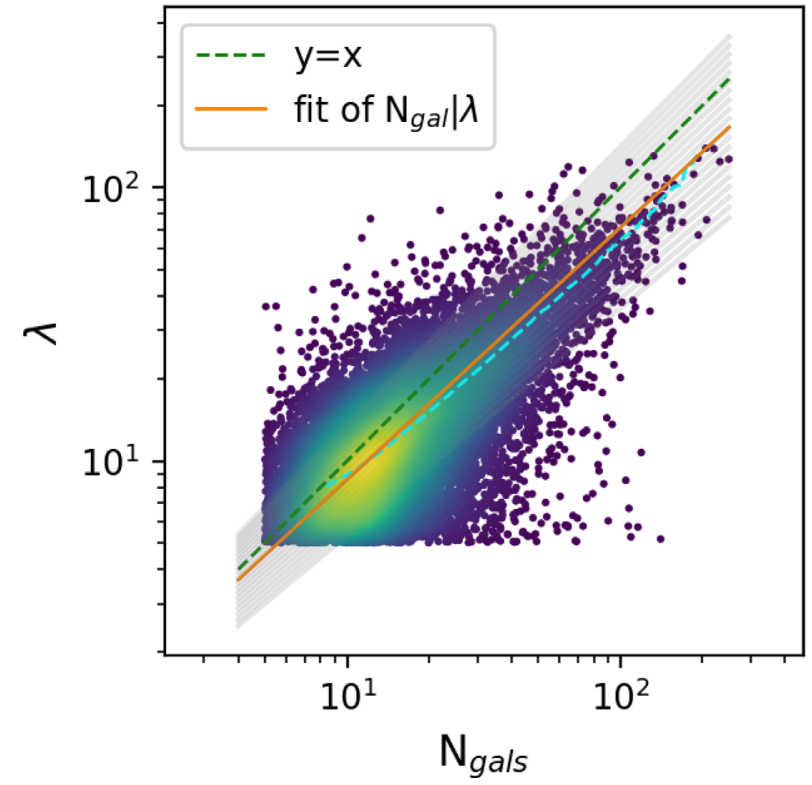

Figure 18. Comparison of richnesses computed by WaZP and redMaPPer for 13664 two-way matched clusters with redshifts in the range $0.1-0.6$, with same centring and redshift offsets $\leq 0.02(1+z)$. The orange line is a powerlaw fit of the richness relation. The dashed blue line represents the thresholds to be applied to the two richnesses to obtain the same cluster densities in the two cluster samples independently from any matching.

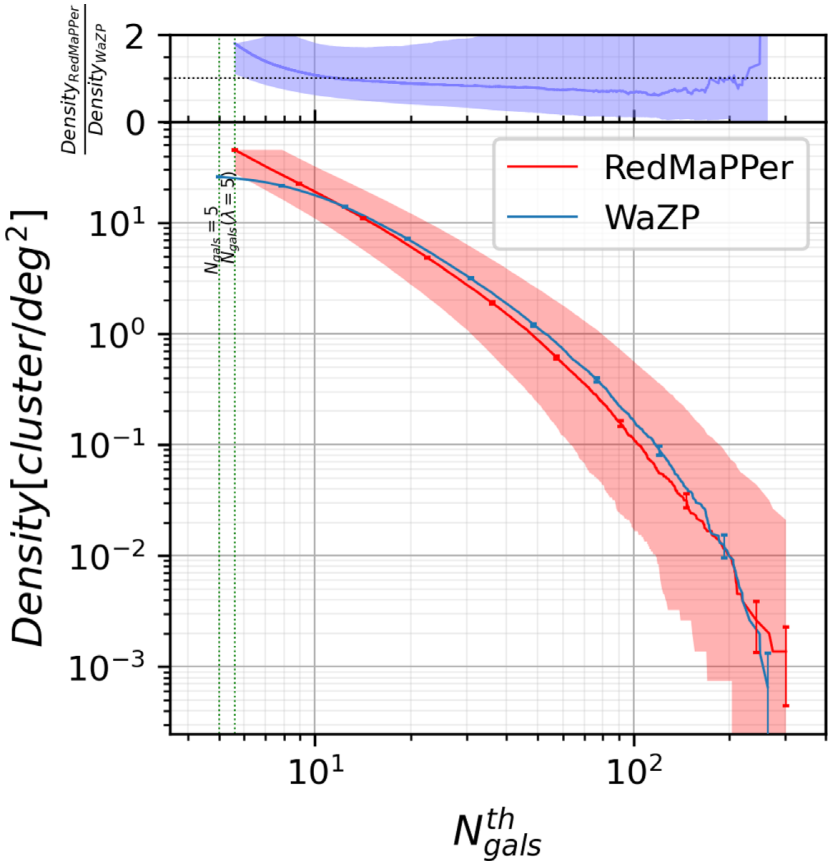

Figure 19. Density of WaZP and redMaPPer catalogues as a function of $N_{\text {gals }}$ threshold. The thresholds for redMaPPer were computed by converting $\lambda$ to $N_{\text {gals }}$ using equation (1), with the shaded regions being the uncertainties propagated and error bars from Poisson noise. The top panel shows the ratio between the densities. 

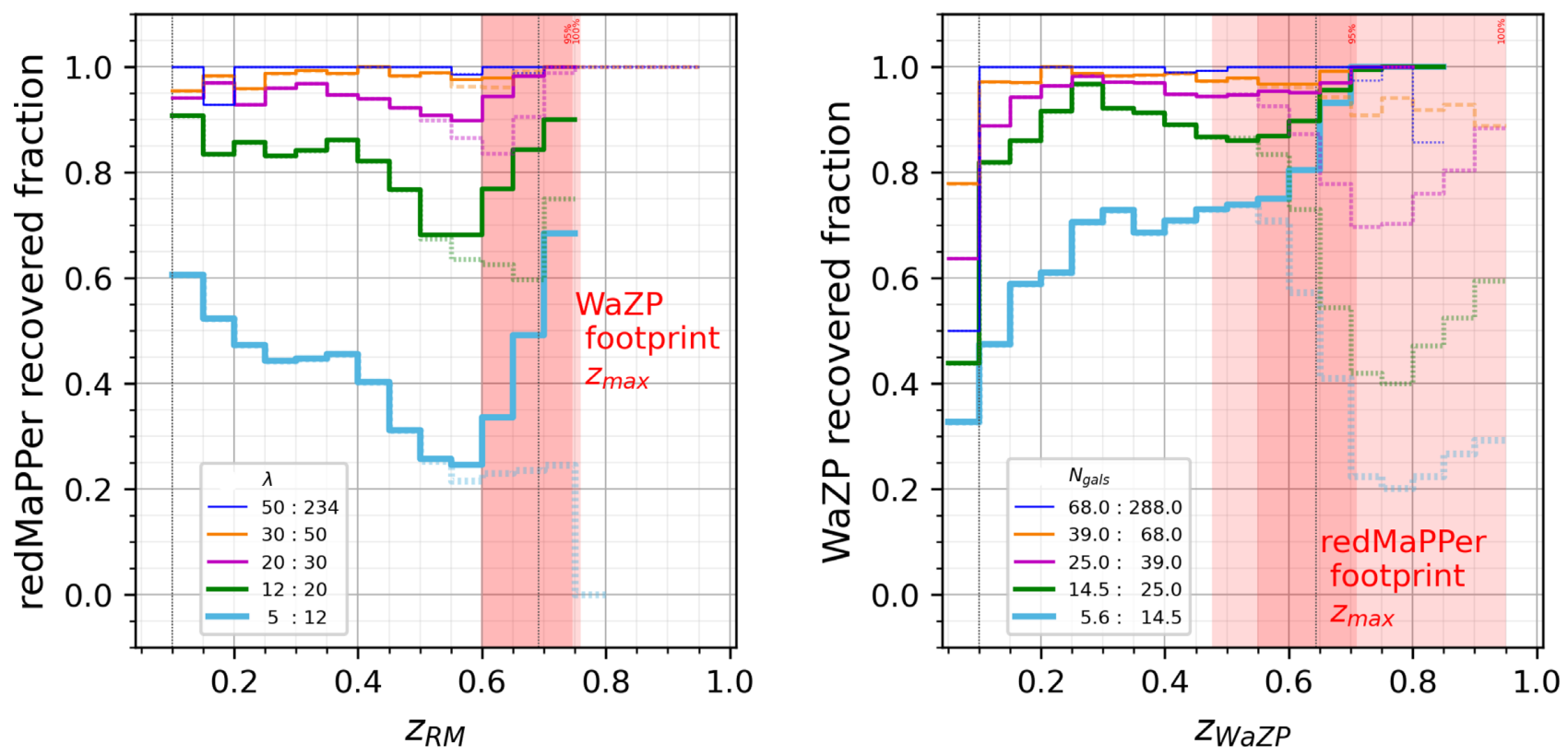

Figure 20. Fraction of clusters detected by both redMaPPer and WaZP binned by richness as a function of redshift. The dotted line is the minimum redshift of the redMaPPer catalogue, the grey shaded area is the region where redMaPPer footprint decreases due its local $z_{\max }$ and the dashed line is the mean of $z_{\max }$. The solid lines are the recovery rates for the matching with redMaPPer volume limited catalogue, and the light shaded lines correspond to matching with redMaPPer volume limited plus redMaPPer full $(\lambda \geq 20)$ catalogue. On the left redMaPPer is used as the reference catalogue (binning by $\lambda$ ) and on the right WaZP is the reference catalogue (binning by $N_{\text {gals }}$ ).

counterpart, respectively, from our recovery rate analysis. These clusters certainly contain information regarding each cluster finder selection function and limitations, however the study of these objects require a different analysis on an object-by-object case and will be done in a future work.

With these considerations, for one-way matching including the possibility of multiple associations, the recovery rate analysis is based on the total of 66955 redMaPPer and 43980 WaZP clusters with 32467 and 33498 matched, respectively.

The recovery rate for each catalogue as a function of redshift in different richness bins is shown in Fig. 20. The left-hand panel shows the fraction of redMaPPer clusters recovered by WaZP and the righthand panel the other way around. The grey shaded area is the redshift region where the redMaPPer (left-hand panel) and WaZP (right-hand panel) footprints decreases in size, with the dashed line being the median value of this footprint redshift limitation (i.e. where the area drops to 50 per cent of the total footprint). The different shades correspond to the 95 per cent and 100 per cent percentiles of the $z_{\max }$ distribution. We binned redMaPPer clusters into 5 samples, 2 bins for lower richness $(\lambda<20)$ clusters, that were not used for cosmological constraints (DES Collaboration 2020), and 3 sample with higher richness. WaZP clusters were binned on the corresponding richness using our fit on equation (1). One can see that redMaPPer clusters with $\lambda \geq 20$ are mostly ( $\geq 90$ per cent) recovered up to the WaZP redshift limit. Similarly, at the same level of richness $\left(N_{\text {gals }} \geq 25\right)$, we find that WaZP clusters are also recovered at more than 90 per cent up to the redMaPPer redshift limit of $\sim 0.7$. We also note rapid increase in the recovery rate of clusters with richness in both cases. Considering the $0.1<z<0.7$ range, the overall recovery rate of redMaPPer clusters is 93.3 per cent, 98.4 per cent, 99.7 per cent for $\lambda \geq 20$ bins (20-30, 30-50, and 50-234, respectively). For WaZP clusters, similarly, we have 95.4 per cent, 97.9 per cent, and 99.7 per cent for $N_{\text {gals }} \geq 25$ bins (25-39, 39-68, and 68-288, respectively). Major differences occur when considering clusters less rich than $\lambda \sim 20$
$\left(N_{\text {gals }} \sim 25\right)$. It is remarkable that even in the $\lambda$ range 5 to $20\left(N_{\text {gals }} 5.6\right.$ to 25$)$, clusters are still recovered at rates between 50 and 60 per cent, depending on the redshift. The dotted vertical line corresponds to the minimum redshift of redMaPPer clusters $(z=0.1)$, hence the low recovery rate for WaZP clusters in the first redshift bin.

We note that the recovery rates reach 100 per cent at high redshifts in both panels of Fig. 20. This is an effect of the footprints variable redshift limit leading to smaller effective area as redshift increases, with only 3 per cent ( 2 per cent) of the redMaPPer (WaZP) area remaining at $z=0.7(0.76)$. Consequently, at those redshifts, all unmatched clusters are removed from the analysis, resulting in an artificially perfect recovery. To obtain a more relevant WaZP recovery rate at high redshifts, we combined redMaPPer volume limited catalogue $(\lambda \geq 5)$ with the redMaPPer full catalogue (uniform $z_{\max }$ of 0.97 and $\lambda \geq 20$ ) and matched it to the WaZP catalogue using the procedure described above. These results are represented by the shaded lines in Fig. 20. We see now that the redMaPPer recovery rate for $\lambda \geq 20$ clusters extends to higher redshifts. Comparing the WaZP recovery fraction to the matching with redMaPPer volume limited only, we have a general decrease at high redshifts. The lower recovery rate of $N_{\text {gals }} \leq 25$ clusters is directly correlated with poorer clusters $(\lambda \leq 20)$ missing in the redMaPPer full catalogue. Richer clusters $\left(N_{\text {gals }} \geq 25\right)$ are affected by the scatter down of the richness relation between both cluster finders.

We conclude from this analysis that, statistically, rich systems are found by both cluster finders, independent of their redshifts, with very few individual differences that are investigated in the next section.

\section{DISCUSSION}

We have shown that all SZ clusters with redshifts $\leq 0.76$ intersecting our footprint are recovered by WaZP cluster finder applied on DES-Y1 data. We have also shown that more than 90 per cent 


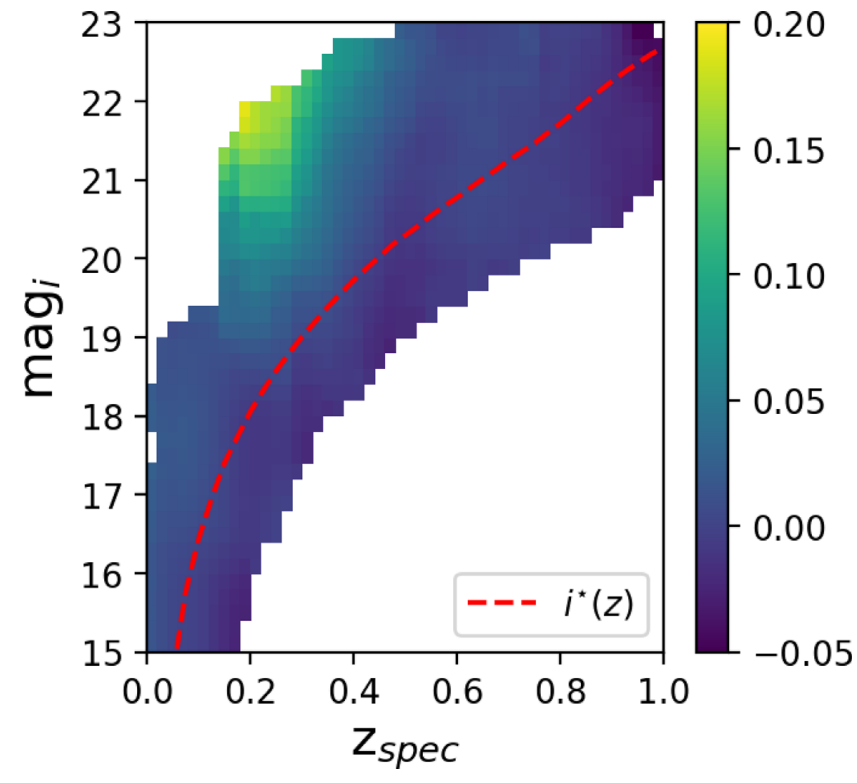

Figure 21. Bias of the galaxy DNF photometric redshifts relative to spectroscopic redshifts as a function of spectroscopic redshift and magnitude. Computation is based here on the SPT region. We require a minimum of 100 spectroscopic redshifts at each redshift - magnitude position. Pixels not satisfying this condition remain white on these maps.

of clusters with richnesses above $\sim 20$ detected by redMaPPer (or 25 by WaZP) are matched to those detected by WaZP (or by redMaPPer). In this section, we compare some properties of the two optical cluster samples. In particular, we explore differences such as redshift discrepancies, overmerging/fragmentation, and the reasons for unmatched systems on both sides.

\subsection{Redshift discrepancies between WaZP and redMaPPer}

Whereas the comparison of WaZP redshifts with spectroscopic redshifts assigned to SZ clusters (see Table 2) showed moderate bias and small scatter, the comparison with redMaPPer clusters revealed stronger discrepancies. This is related to the fact that redMaPPer (and WaZP) clusters are on average much less massive than SPT clusters. This is confirmed if we restrict the cross-match between WaZP and redMaPPer to richer clusters. In that case, as shown in the right-hand panel of Fig. 17, a reduced redshift bias and scatter is observed between the two samples.

The fraction of redshift outliers (defined by a redshift difference $\geq 3 \sigma_{z}(1+z)$ ) is less than 5 per cent of our clusters, with 78 per cent (82 per cent) of them having $N_{\text {gals }} \leq 25(\lambda \leq 20)$. These redshift outliers are mainly produced at $z_{\mathrm{W} a \mathrm{ZP}} \sim 0.4$. This also reflects on WaZP cluster number counts (Fig. 9), that showed a peak at redshift $\sim 0.4$ that becomes more prominent when considering the poorest clusters. From the same cluster number counts, a deficit of clusters at $z \sim 0.3$ can also be noticed. These points support the idea that in the redshift range $0.15-0.35$, WaZP detects on average the same clusters as redMaPPer but shifts a fraction of these to $z \sim 0.4$.

From global statistics of photometric redshifts, only moderate bias is measured (see right-hand panel of Fig. 5). However, this global bias includes galaxies of all magnitudes down to $m_{i}^{*}+1.5$. In order to understand the shift in redshift of a fraction of WaZP clusters, one needs to investigate the photometric redshift bias at least as a function of both redshift and magnitude. This is what is shown in Fig. 21. We binned our spectroscopic sample in $i$-band magnitude and spectroscopic redshift and computed, for each bin with at least 100 galaxies, the median and standard deviation of $\left(z_{\text {phot }}-z_{\text {spec }}\right) /(1$ $\left.+z_{\text {spec }}\right)$. The amplitude of the photometric redshift bias is shown as a colour code. In most regions of this diagram, the bias is moderate, consistent with the global bias. However, for redshifts between 0.15 and 0.35 and $i$-band magnitudes fainter than $\sim 20$, we find a strong bias that reaches values of $0.1-0.2$.

The origin of this strong bias is two-folded. It is first due to the lack of $u$-band and to the transition of the $4000 \AA$ break between $g$ and $r$ band at redshift $z \sim 0.3-0.4$. Secondly, it is due to the lack of faint $\left(m_{i} \geq 20\right)$ red galaxies at redshifts below $\sim 0.35$ in our spectroscopic training sample. We stress that for these faint, lowredshift and red galaxies, the bias may be even larger as it cannot be estimated properly. The consequence is that their photometric redshift is overestimated, pushed to redshifts where the training set samples better the same (magnitude, colours) space. This effect has already been stressed in several other studies (e.g. fig. 25 of Rykoff et al. 2016).

This statement can actually be tested by comparing how a DES redshift biased WaZP cluster is detected in the Sloan Digital Sky Survey, that is covered in $u, g, r, i, z$ bands in the overlapping Stripe 82 region. As an example, we selected from our DES-Y1-S82 run, one cluster of richness above 60 with a redshift 0.43 , whereas the same cluster is detected by redMaPPer at a redshift of 0.28 . WaZP was run on a small section of SDSS-S82 around that cluster with the same settings but based on SDSS DR-12 photometric redshifts from Beck et al. (2016). Based on these redshifts, WaZP recovers a much lower redshift $(z=0.28)$ for the cluster, which is consistent with redMaPPer and with the available BCG spectroscopic redshift. In Fig. 22, we show the redshifts and magnitudes of galaxies classified as cluster members for the two detections, based on DES and on SDSS. One can first notice a large overlap between the members. Then, one can clearly see that these common members are systematically shifted to larger redshifts within the DES. As this effect is stronger for fainter objects, one can also notice for instance that the BCG, at a magnitude of 16.5, has an unbiased redshift. The consequence is that the BCG was not considered as a member in the DES based membership. To conclude, WaZP based on DNF-DES photometric redshifts seems able to recover clusters at $z \sim 0.3$, but redshift, membership and therefore richness may be severely affected.

New approaches are currently being investigated to correct for the impact on cluster detection and characterization of the photometric redshift bias effect.

\subsection{Relative fragmentation}

In the previous section, the relative completeness of the two optical cluster finders presented in Fig. 20 is meant to highlight the fraction of clusters without any counterpart. For those clusters tagged as matched, this matching does not assure a one-to-one correspondence for the matched clusters, but only that a cluster from one sample has at least one counterpart in the matched sample. Here, we examine clusters from one sample that are matched to more than one cluster in the opposite sample. In the case of a two-way match, the richest counterparts are selected letting the additional possibilities unmatched. The extra component(s) involved in the one-way match only could be interpreted as a cluster substructures in the other sample, or as a missed cluster, depending on the adopted definition of each cluster finder.

In terms of one-way matching, from Fig. 20, we found that 96 per cent of WaZP clusters in the redshift range $0.1-0.6$ with $N_{\text {gals }}$ $\geq 25$ have a redMaPPer counterpart, and conversely, 94 per cent of 


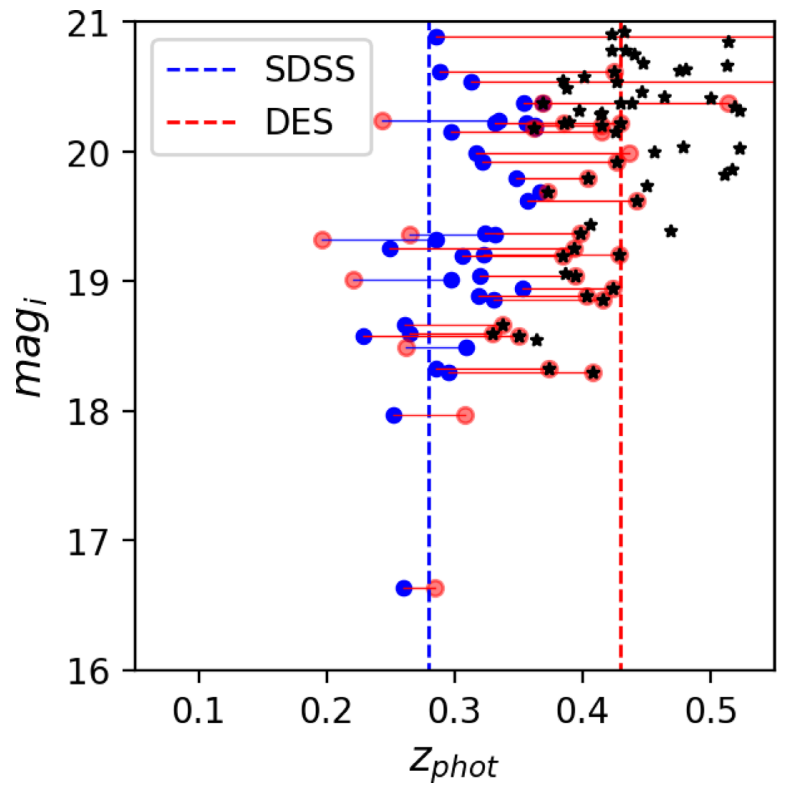

Figure 22. This figure illustrates how the bias in photometric redshifts shown statistically in right-hand panel of Fig. 5 may affect cluster members at $z \sim$ 0.3 . It displays the members of a galaxy cluster detected in Y1-S82 by WaZP at redshift $z=0.43$ (black stars). This cluster is also found by redMaPPer but with a redshift $z=0.28$. When using SDSS photometry WaZP recovers the cluster at the same redshift as redMaPPer (blue dashed line), and associated members are shown in blue. These SDSS based members have a different photometric redshift when computed with DNF based on DES photometry (i.e. without the $u$-band). The latter are shown in red, and are linked to a blue point when it is the same galaxy. The common red and black symbols show that the two detections have many members in common. However, the BCG (at magnitude 16.5), for instance, was missed as its redshift is not biased. As the DNF+DES-Y1 bias is strongly magnitude dependent, it pulls members apart in two different redshift bins.

redMaPPer clusters with $\lambda \geq 20$ have a WaZP counterpart in the same redshift range. If we now consider two-way matches, only 87 per cent of WaZP clusters have a redMaPPer counterpart, whereas 91 per cent of redMaPPer clusters are two-way matched, about the same fraction as for the one-way matching. The larger decrease of matches for WaZP clusters when going from one to two-way matching suggests that they are in average relatively more fragmented (or redMaPPer clusters relatively more merged). This is what we investigate below.

The apparent larger fragmentation of WaZP clusters could be due to the presence of very low richness clusters in the periphery of richer ones. To test this, we evaluated the relative fragmentation rate of the two cluster finders considering different richness cuts in the associated systems. The relative fragmentation rate is estimated as the fraction of matches that have more than one counterpart. If we start from WaZP clusters with $N_{\text {gals }} \geq 25$, the relative fragmentation rate is 22 per cent, 6 per cent, and 2 per cent when considering counterparts with, respectively, $\lambda \geq 5,10,20$. Conversely, starting from redMaPPer clusters with $\lambda \geq 20$, the relative fragmentation rate is 30 per cent, 24 per cent, and 14 per cent when considering counterparts with, respectively, $N_{\text {gals }} \geq 6,12,25$. The ratio (WaZP to redMaPPer) of the fragmentation rates increases strongly when considering richer multiple counterparts. We can conclude from this that WaZP tends to find pairs of relatively rich clusters more frequently than redMaPPer.

Should multiple systems be seen as one or several clusters is a matter of cluster definition for each cluster finder. They may also suggest a wrong tuning of the detection algorithm leading to undesirable fragmentation within a clearly unique cluster. To address this point, we visually inspected the 50 richest WaZP cluster pairs and found that the vast majority do correspond to clear separate groups. In very few cases only WaZP detected two peaks clearly within the same cluster. In Fig. 23, we show two relatively rich cases at redshifts 0.38 and 0.68 . In both cases, the redMaPPer cluster radii are only slightly larger than the distance between the two WaZP clusters, which assured the one way matching of both WaZP systems. It is likely here that the galaxies from the extra WaZP clusters were percolated to the most likely redMaPPer cluster reducing their weight as members of a secondary cluster and eventually leading to only one detection (Rykoff et al. 2014; Section 9). However, let us stress that the detection algorithms may be tuned to find different overdensities, leading to different samples with their own selection function.

\subsection{Unmatched systems}

Let us now turn to the WaZP or redMaPPer clusters for which no counterpart was found using our one-way matching procedure. Our goal here is to provide some insight on the reasons why some systems, or types of systems would not be detected by one algorithm or the other. We should first stress that our matching procedure is designed in such a way that we have strongly limited the number of unmatched clusters that could be due e.g. to edge effects, variable depths of the used reference bands, or differences in estimated redshifts.

Treating edge effects properly appeared to be a critical issue as it concerns a significant number of detections due to the complex geometry of the masked regions. Moreover each cluster sample was not built using exactly the same footprint, in particular due to the different reference band used. We considered regions covered by the two footprints, and also followed redMaPPer's prescription and discarded clusters that would intersect empty regions of the galaxy catalogue by more than 20 per cent within a $1 \mathrm{Mpc}$ radius. Note that this area fraction is actually weighted by a projected NFW profile as described in Rykoff et al. (2012). Concerning the adopted tolerance in redshift difference, as shown above, we have carried out an empirical approach, precisely to avoid unmatched systems that would be detected on both sides but with a too large redshift discrepancy. This case may still happen in our matched catalogue, but with a lower occurrence.

In order to qualify the unmatched clusters, we have carried out a visual inspection of the 60 richest ones (for each cluster finder) in the redshift range 0.1 to 0.65 . These systems have richnesses $N_{\text {gals }} \geq$ 30 and $\lambda \geq 25$.

Without trying to derive precise statistics from this inspection, unmatched systems clearly enter two categories common to the two cluster finders. The first one, corresponding to one third of the inspected systems, is made of clear concentrated overdensities of red galaxies (two examples are shown in Fig. 24, one detected by WaZP and the second by redMaPPer). For these systems, possible edge or depth effects were checked and discarded. Those not found by redMaPPer have redshifts ranging uniformly from 0.3 to 0.6 , whereas those not found by WaZP are concentrated in two redshift bins, around $0.25-0.35$ (possibly due to the photometric redshift bias described above) and the second around 0.5-0.6.

A second category covering more than half of the inspected clusters is composed of much looser systems, without any obvious central concentration, sometimes possibly fragments of larger scale filamentary structures, and in some few cases no apparent cluster at all. These loose systems may appear as poorer clusters even though they are selected among the richest unmatched, typically $\lambda$ (or $N_{\text {gals }}$ ) 

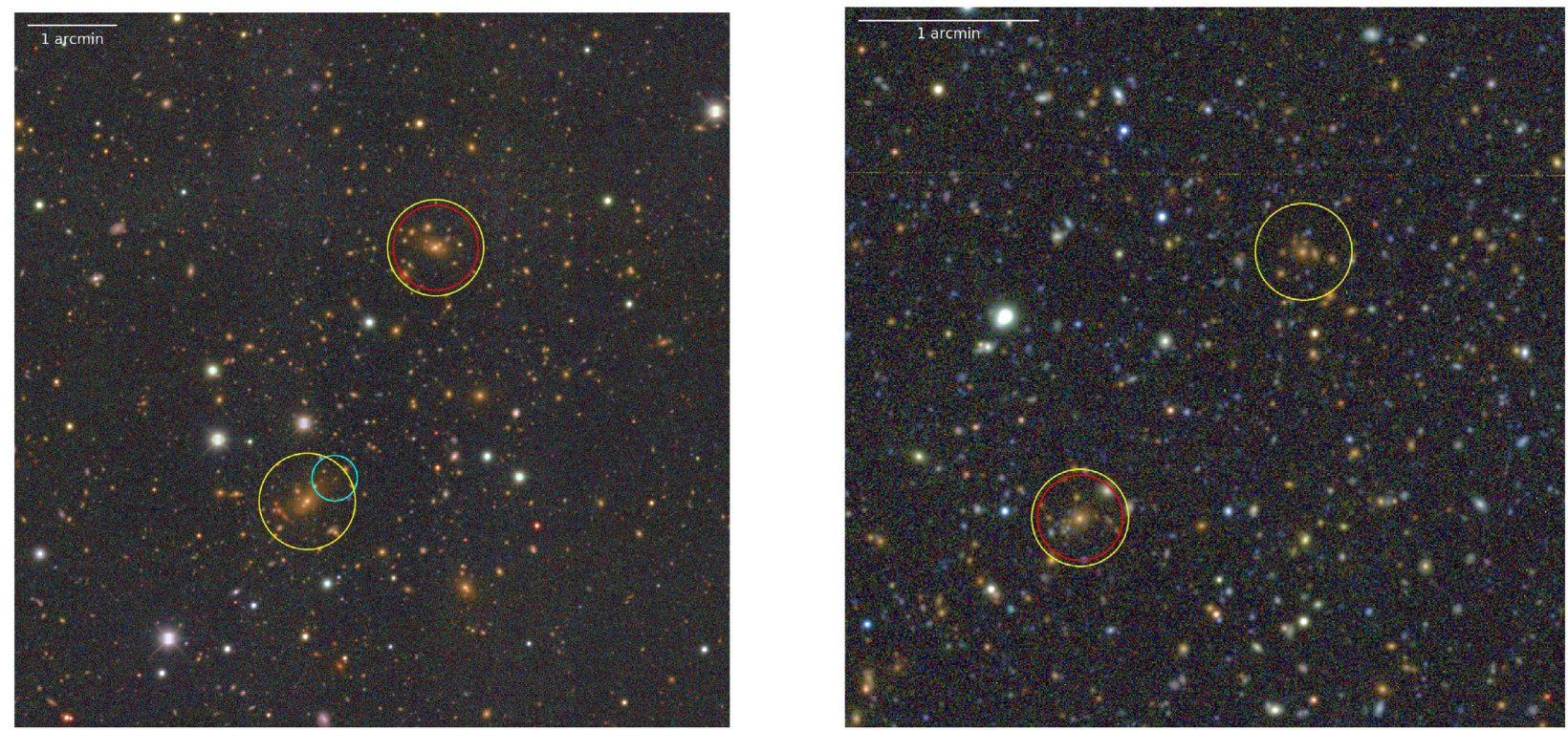

Figure 23. Examples of two clusters that are one-way matched but not two-way matched. In these examples, WaZP detects two clusters (yellow circles), whereas redMaPPer detects one (red circle), with a cluster radius reaching the second component. In the left-hand panel $(\mathrm{RA}=34.312, \mathrm{Dec}=-52.760)$, WaZP detected two clusters at $z=0.39 / 0.38$ separated by $1 \mathrm{Mpc}$. The top right detection is a redMaPPer cluster with $z=0.34\left(\lambda=119\right.$ and $\left.N_{\text {gals }}=118\right)$. The bottom left detection $\left(N_{\text {gals }}=81\right)$ also matches an SPT detection (cyan circle). In the right-hand panel (RA $=28.125$, Dec $\left.=-40.944\right)$, WaZP detected two clusters at $z=0.67 / 0.69$ separated by $1 \mathrm{Mpc}$. The bottom left detection is a redMaPPer cluster with $z=0.68\left(\lambda=32\right.$ and $\left.N_{\text {gals }}=47\right)$. The top right detection has a richness $N_{\text {gals }}=66$.
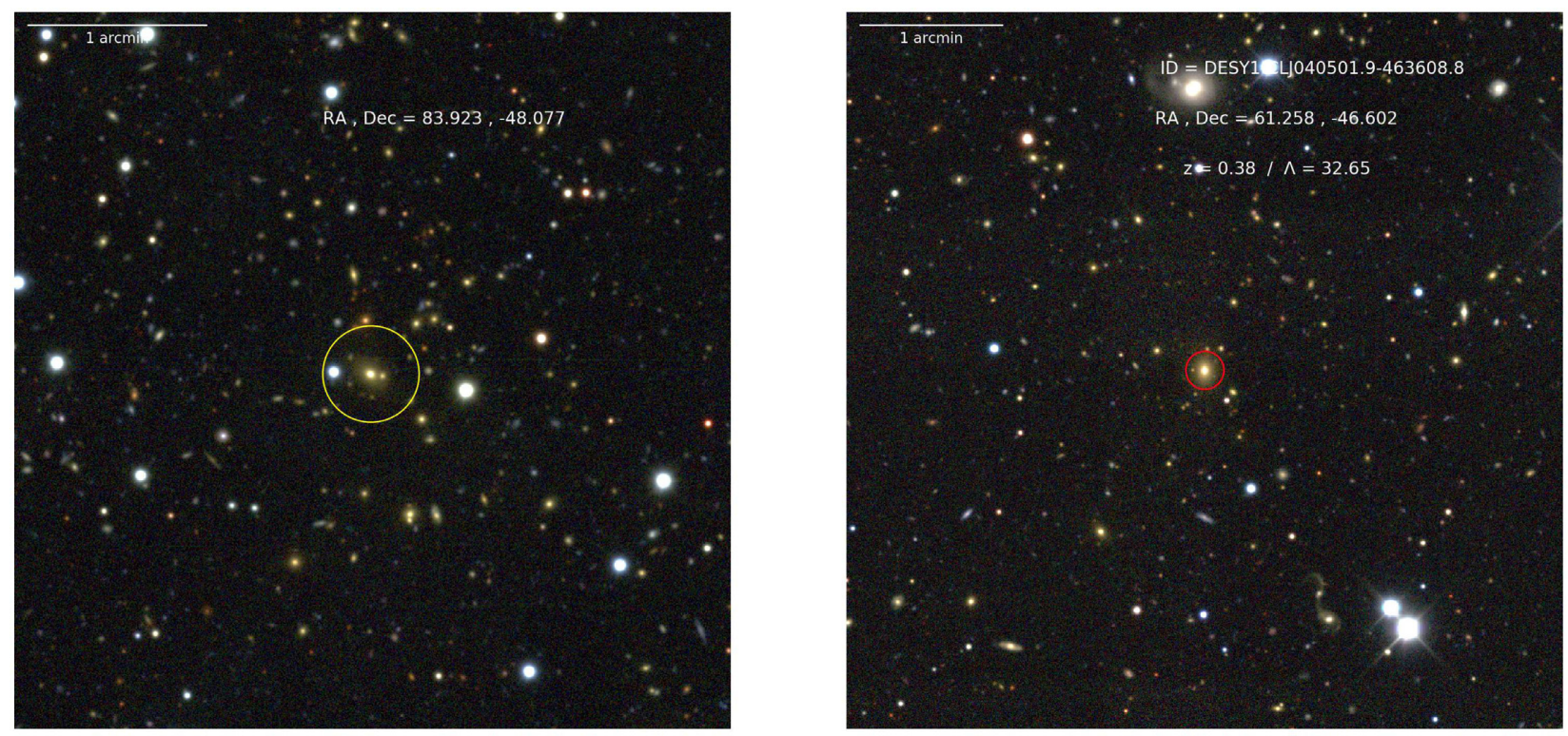

Figure 24. Examples of a WaZP cluster (left-hand panel, yellow circle) and a redMaPPer cluster (right-hand panel, red circle) both tagged as unmatched. The redshifts and richnesses of these two clusters are $z=0.44 / N_{\text {gals }}=56$. (left-hand panel) and $z=0.38 / \lambda=32$ (right-hand panel).

30-35. One typical example is shown in Fig. 25 where we compare the case of two redMaPPer clusters at the same redshift $(z \sim 0.6)$ and with similar richnesses $(\lambda \sim 30)$. The concentrated system is well recovered by WaZP at the same redshift and with similar richness, whereas no counterpart was found for the looser one. Similar opposite situations occur when considering WaZP clusters as a reference.

What seems to be common to these loose unmatched clusters is that they are often characterized, at a given richness, by a lower SNR (in the case of WaZP) and a lower likelihood (in the case of redMaPPer). In order to verify this observation statistically, we have compared the WaZP SNR and redMaPPer likelihood of the matched and unmatched clusters. To do this, as these two quantities depend in average on both redshift and richness, we have computed the median and 68 percentile of the SNR and likelihood in bins of redshift and richness. We can then compare how each matched and unmatched cluster deviates relative to its local (in redshift-richness space) median SNR or likelihood. The result, considering all clusters in the redshift range $0.1-0.6$ and with $N_{\text {gals }} \geq 25$ and $\lambda \geq 20$, is shown in Fig. 26. Clearly, unmatched clusters have in average lower SNR 

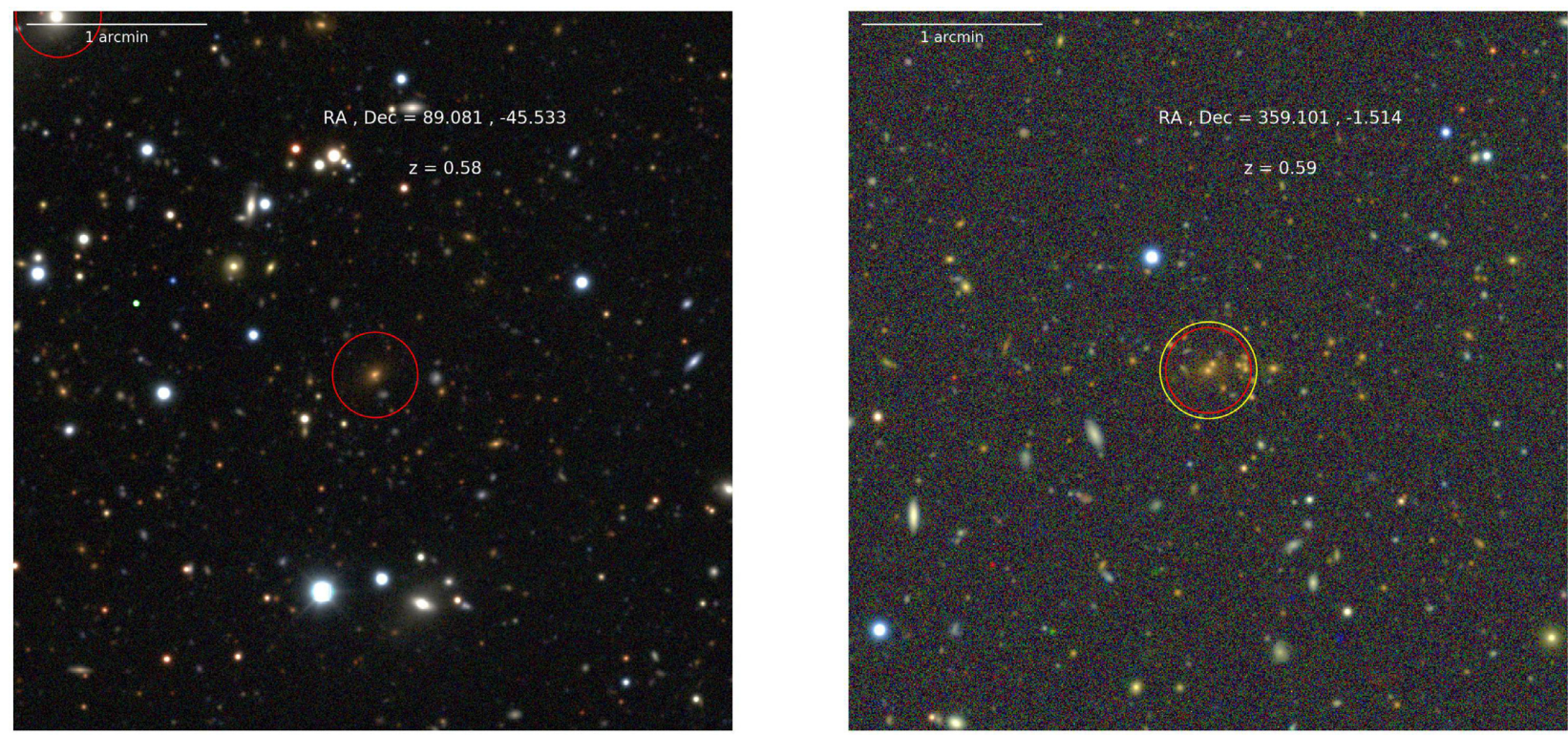

Figure 25. Example of a redMaPPer cluster (red circle) not found by WaZP (left-hand panel) compared to a redMaPPer cluster with same richness and redshift that is matched to WaZP (right-hand panel, yellow circle). The two redMaPPer clusters have a richness $\lambda=30$, and a redshift $z=0.58-0.59$. The matched WaZP cluster was found at $z=0.6$ with a richness $N_{\text {gals }}=37$.
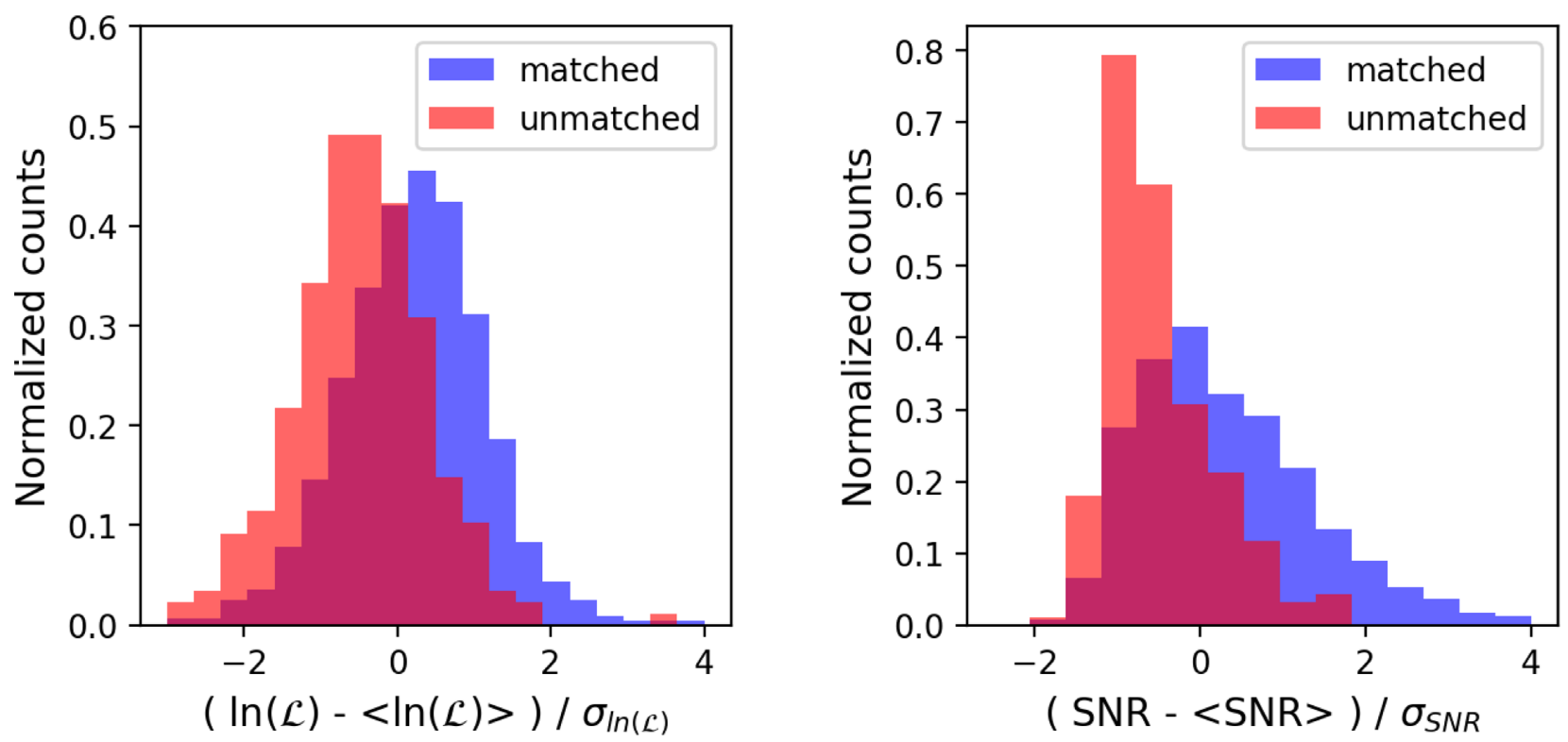

Figure 26. Comparison of the distributions of redMaPPer cluster likelihoods relative to the median likelihood at the clusters redshift and richness (left-hand panel) and WaZP cluster SNRs relative to the median SNR at the clusters redshift and richness (right-hand panel) for matched (blue) and unmatched (red) clusters. For both samples, we have considered here clusters with richnesses $\lambda \geq 20$ and $N_{\text {gals }} \geq 25$ and redshifts in the range $0.1-0.6$. Note that the distributions are normalized; in both cases, the unmatched clusters are $\sim 20$ times less numerous than the matched ones.

or lower likelihood than the average, suggesting that these quantities should be considered in the cluster selection function.

\section{CONCLUSIONS}

In this paper, we present WaZP a new $(2+1) \mathrm{D}$ cluster finder based on photometric redshifts. It is applied to DES-Y1A1 data and resulting samples are compared to those derived from the SPT survey based on the SZ effect, and from the redMaPPer cluster finder applied to the same photometric data.
Our conclusions can be listed as follows.

(i) A galaxy Value Added Catalogue was derived from the DES Y1A1 survey. It is controlled by the $i$-band that is used for stargalaxy separation and for determining the local depth. Depending on the location, complete galaxy samples can be built down to $m_{i}=$ 22.25-23, with a median (corresponding to half of the survey area) completeness magnitude limit of $m_{i}=22.7$.

(ii) The WaZP cluster finder was applied to DES Y1A1 survey led to the detection of 60547 clusters over $1511.13 \mathrm{deg}^{2}$, with redshifts ranging from 0.05 to 0.9 and richness greater than 5. Due to the $i$-band 
limiting magnitude of the survey and the adopted limiting magnitude for estimating richnesses, complete richnesses are derived for clusters in the redshift range $0.05-0.60$. Clusters detected at larger redshifts get increasingly incomplete richnesses depending on the local depth.

(iii) Considering the SPT cluster sample intersecting the DES Y1A1 footprint in the redshift range 0.05-0.76, WaZP was shown to recover all $293 \mathrm{SZ}$ clusters. Comparing redshifts of both cluster finders, we found a bias of 0.012 and a scatter of the same order of redshift uncertainties $(\sim 0.025)$. When we restrict to SZ clusters with an assigned spectroscopic redshift, all these quantities are lowered by 40 per cent.

(iv) Cross-matching WaZP and redMaPPer catalogue led to the development of an iterative matching algorithm to minimize incorrect associations. Special care was taken to deal with edge effects and depth variations. It resulted in matching 28621 clusters in the twoway criteria with richnesses $N_{\text {gals }}$ (and $\lambda$ ) down to 5 . Considering oneway matching for clusters richer than $N_{\text {gals }}=25(\lambda=20)$ with 0.1 $<z<0.6$, we showed that WaZP recovered 96 per cent redMaPPer clusters, and, symmetrically, redMaPPer recovered 94 per cent WaZP clusters.

(v) The centring offset between WaZP and redMaPPer is less than $200 \mathrm{kpc}$ in most cases (97 per cent), which is much less than the matching criteria used.

(vi) Comparison of the estimated redshifts from redMaPPer and WaZP shows an overall good agreement. However, a fraction of WaZP clusters suffer from a redshift bias, reflecting the underlying galaxy photometric redshift bias. Note that this effect does not seem to prevent detection in general.

(vii) Despite different definitions, the comparison of WaZP and redMaPPer richnesses shows a strong correlation. The scatter of this relation will be analysed in details on a separate paper in a future work. We also computed a richness relation based on thresholds that provided the same densities of clusters. This relation is remarkably close to the fit from matched clusters, supporting the idea that, on average, the ranking of the two cluster samples by their richness is similar.

(viii) The study of the relative fragmentation of WaZP and redMaPPer clusters showed that WaZP tends to find pairs of relatively rich clusters more frequently than redMaPPer.

(ix) The visual inspection of 60 unmatched clusters richer than $N_{\text {gals }} \geq 25$ (for WaZP) and $\lambda \geq 20$ (for redMaPPer), showed that (for both samples) $1 / 3$ of these are clear concentrations of red galaxies, which, in itself could help to improve the completeness of both samples. A second category representing more than half of the inspected unmatched systems is composed of much looser systems or more filamentary structures. This dominant category of unmatched clusters has been shown statistically to be characterized by lower SNRs (for WaZP) and lower likelihoods (for redMaPPer) than for the matched clusters.

One of the main aspects of this work is to address the relative completenesses of two optical cluster finders based on different methods and applied to the same survey. As shown in this paper, the comparison of the resulting samples in itself is not a trivial task free of assumptions. However, it is a useful guide to detect features of cluster finding algorithms when applied to large surveys with all their complexity (missing data, mis-classified sources, depth variation, etc.). In that sense, such comparisons are complementary to those performed with mock catalogues, that can be used, for instance, to address the sample purity in addition to completeness.
A limitation of this study is the quality of the photometric redshifts partly hampered by the poor representation of faint red galaxies in our spectroscopic training set, in particular at low redshifts $(z$ $\leq 0.35$ ). In a companion paper, we are exploring the impact of using a much larger spectroscopic training set, and the use of several photometric redshift codes. We will also study the impact of the star-galaxy classification algorithm. Finally, we will compare WaZP cluster samples based on $i$-band and $z$-band reference magnitudes with a special focus on high redshift cluster detection.

\section{ACKNOWLEDGEMENTS}

MA was supported by FAPESP (2013/26612-2) and CNPq (380101/2017-3, 165049/2017-0, 381135/2018-7). CB acknowledges the support obtained from the Bonus-Qualité-Recherche 2013 \& 2017 Programs of the J.-L. Lagrange Laboratory (UMR 7293).

CB thanks J.-L. Starck for his help in using and configuring the MR/1 multiresolution to optimally build galaxy density maps. $\mathrm{CB}$ also thanks S. Maurogordato, A. Cappi, and E. Slezak for many stimulating discussions during the development of the WaZP cluster finder algorithm.

Funding for the DES Projects has been provided by the U.S. Department of Energy, the U.S. National Science Foundation, the Ministry of Science and Education of Spain, the Science and Technology Facilities Council of the United Kingdom, the Higher Education Funding Council for England, the National Center for Supercomputing Applications at the University of Illinois at Urbana-Champaign, the Kavli Institute of Cosmological Physics at the University of Chicago, the Center for Cosmology and Astro-Particle Physics at the Ohio State University, the Mitchell Institute for Fundamental Physics and Astronomy at Texas A\&M University, Financiadora de Estudos e Projetos, Fundação Carlos Chagas Filho de Amparo à Pesquisa do Estado do Rio de Janeiro, Conselho Nacional de Desenvolvimento Científico e Tecnológico and the Ministério da Ciência, Tecnologia e Inovação, the Deutsche Forschungsgemeinschaft and the Collaborating Institutions in the Dark Energy Survey.

The Collaborating Institutions are Argonne National Laboratory, the University of California at Santa Cruz, the University of Cambridge, Centro de Investigaciones Energéticas, Medioambientales y Tecnológicas-Madrid, the University of Chicago, University College London, the DES-Brazil Consortium, the University of Edinburgh, the Eidgenössische Technische Hochschule (ETH) Zürich, Fermi National Accelerator Laboratory, the University of Illinois at UrbanaChampaign, the Institut de Ciències de l'Espai (IEEC/CSIC), the Institut de Física d'Altes Energies, Lawrence Berkeley National Laboratory, the Ludwig-Maximilians Universität München and the associated Excellence Cluster Universe, the University of Michigan, NFS's NOIRLab, the University of Nottingham, The Ohio State University, the University of Pennsylvania, the University of Portsmouth, SLAC National Accelerator Laboratory, Stanford University, the University of Sussex, Texas A\&M University, and the OzDES Membership Consortium.

Based in part on observations at Cerro Tololo Inter-American Observatory at NSF's NOIRLab (NOIRLab Prop. ID 2012B-0001; PI: J. Frieman), which is managed by the Association of Universities for Research in Astronomy (AURA) under a cooperative agreement with the National Science Foundation.

The DES data management system is supported by the National Science Foundation under Grant Numbers AST-1138766 and AST-1536171. The DES participants from Spanish institutions are partially supported by MICINN under grants ESP201789838, PGC2018-094773, PGC2018-102021, SEV-2016-0588, 
SEV-2016-0597, and MDM-2015-0509, some of which include ERDF funds from the European Union. IFAE is partially funded by the CERCA program of the Generalitat de Catalunya. Research leading to these results has received funding from the European Research Council under the European Union's Seventh Framework Program (FP7/2007-2013) including ERC grant agreements 240672, 291329, and 306478. We acknowledge support from the Brazilian Instituto Nacional de Ciência e Tecnologia (INCT) do e-Universo (CNPq grant 465376/2014-2).

This manuscript has been authored by Fermi Research Alliance, LLC under Contract No. DE-AC02-07CH11359 with the U.S. Department of Energy, Office of Science, Office of High Energy Physics.

\section{DATA AVAILABILITY}

The WaZP DES-Y1A1 cluster products with a full description of the catalogues and columns can be found are available in its online supplementary material ${ }^{5}$.

\section{REFERENCES}

Abbott T. M. C. et al., 2018, ApJS, 239, 18

Adami C., Mazure A., Katgert P., Biviano A., 1998, A\&A, 336, 63

Adami C. et al., 2010, A\&A, 509, A81

Adami C. et al., 2018, A\&A, 620, A5

Aguena M., Lima M., 2018, Phys. Rev. D, 98, 123529

Aihara H. et al., 2011, ApJS, 193, 29

Arnouts S. et al., 2001, A\&A, 379, 740

Ascaso B., Mei S., Bartlett J. G., Benítez N., 2017, MNRAS, 464, 2270

Beck R., Dobos L., Budavári T., Szalay A. S., Csabai I., 2016, MNRAS, 460, 1371

Bellagamba F., Roncarelli M., Maturi M., Moscardini L., 2018, MNRAS, 473, 5221

Benson B. A. et al., 2013, ApJ, 763, 147

Bertin E., Arnouts S., 1996, A\&AS, 117, 393

Bleem L. E. et al., 2015, ApJS, 216, 27

Bleem L. E. et al., 2020, ApJS, 247, 25

Bocquet S. et al., 2019, ApJ, 878, 55

Botzler C. S., Snigula J., Bender R., Hopp U., 2004, MNRAS, 349, 425

Bouy H., Bertin E., Moraux E., Cuillandre J. C., Bouvier J., Barrado D., Solano E., Bayo A., 2013, A\&A, 554, A101

Brodwin M. et al., 2013, ApJ, 779, 138

Capak P. et al., 2004, AJ, 127, 180

Capak P. et al., 2007, ApJS, 172, 99

Carlstrom J. E. et al., 2011, PASP, 123, 568

Castignani G., Benoist C., 2016, A\&A, 595, A111

Chiu I. et al., 2018, MNRAS, 478, 3072

Costanzi M., Rozo E. et al., 2019, MNRAS, 482, 490

Cucciati O. et al., 2010, A\&A, 520, A42

Dark Energy Survey Collaboration, 2016, MNRAS, 460, 1270

de Jong J. T. A., Verdoes Kleijn G. A., Kuijken K. H., Valentijn E. A., 2013, Exp. Astron., 35, 25

De Vicente J., Sánchez E., Sevilla-Noarbe I., 2016, MNRAS, 459, 3078

DeRose J. et al., 2019, preprint (arXiv:1901.02401)

DES Collaboration, 2020, Phys. Rev. D, 102, 023509

Desai S. et al., 2012, ApJ, 757, 83

Diehl H. T. et al., 2016, in AlisonP.,ed., Observatory Operations: Strategies, Processes, and Systems VI, SPIE, Edinburgh, United Kingdom, p. 99101D

Drlica-Wagner A. et al., 2018, ApJS, 235, 33

Euclid Collaboration, 2019, A\&A, 627, A23

\footnotetext{
${ }^{5}$ https://www.linea.gov.br/catalogs/wazp/
}

Fausti Neto A. et al., 2018, Astron. Comput., 24, 52

Fioc M., Rocca-Volmerange B., 1997, A\&A, 326, 950

Flaugher B., 2005, Int. J. Mod. Phys. A, 20, 3121

Flaugher B. et al., 2015, AJ, 150, 150

George M. R. et al., 2011, ApJ, 742, 125

Gerke B. F. et al., 2005, ApJ, 625, 6

Gerke B. F. et al., 2012, ApJ, 751, 50

Gladders M. D., Yee H. K. C., 2000, AJ, 120, 2148

Górski K. M., Hivon E., Banday A. J., Wandelt B. D., Hansen F. K., Reinecke M., Bartelmann M., 2005, ApJ, 622, 759

Goto T. et al., 2002, AJ, 123, 1807

Gschwend J. et al., 2018, Astron. Comput., 25, 58

Hansen S. M., McKay T. A., Wechsler R. H., Annis J., Sheldon E. S., Kimball A., 2005, ApJ, 633, 122

Hao J. et al., 2010, ApJS, 191, 254

Harris W. E., 2010, preprint (arXiv:1012.3224)

Ilbert O. et al., 2006, A\&A, 457, 841

Kaiser N. et al., 2002, in Tyson J. A., Wolff S., eds, Proc. SPIE Conf. Ser. Vol. 4836, Survey and Other Telescope Technologies and Discoveries. SPIE, Bellingham, p. 154

Kim R. S. J. et al., 2002, AJ, 123, 20

Kim M. et al., 2007, ApJS, 169, 401

Knobel C. et al., 2009, ApJ, 697, 1842

Koester B. P. et al., 2007, ApJ, 660, 239

Laureijs R. et al., 2011, preprint (arXiv:1110.3193)

Leistedt B. et al., 2016, ApJS, 226, 24

Licitra R., Mei S., Raichoor A., Erben T., Hildebrandt H., 2016, MNRAS, 455,3020

Lima M., Hu W., 2005, Phys. Rev. D, 72, 043006

Lin Y.-T., Mohr J. J., Gonzalez A. H., Stanford S. A., 2006, ApJ, 650, L99

Lloyd-Davies E. J. et al., 2011, MNRAS, 418, 14

LSST Science Collaboration, 2009, preprint (arXiv:0912.0201)

Lumsden S. L., Nichol R. C., Collins C. A., Guzzo L., 1992, MNRAS, 258, 1

Mantz A., Allen S. W., Rapetti D., Ebeling H., 2010, MNRAS, 406, 1759

McClintock T. et al., 2019, MNRAS, 482, 1352

Mei S. et al., 2009, ApJ, 690, 42

Melchior P. et al., 2015, MNRAS, 449, 2219

Melchior P. et al., 2017, MNRAS, 469, 4899

Morganson E., Gruendl R. A., Menanteau F., DES Collaboration, 2018, PASP, 130, 074501

Murphy D. N. A., Geach J. E., Bower R. G., 2012, MNRAS, 420, 1861

Neilsen Eric H. J., Annis J. T., Diehl H. T., Swanson M. E. C., D’Andrea C., Kent S., Drlica-Wagner A., 2019, preprint (arXiv:1912.06254)

Olsen L. F. et al., 1999, A\&A, 345, 363

Olsen L. F. et al., 2008, A\&A, 478, 93

Palmese A. et al., 2016, MNRAS, 463, 1486

Pereira M. E. S. et al., 2018, MNRAS, 474, 1361

Pereira M. E. S. et al., 2020, MNRAS, 498, 5450

Planck Collaboration XIII, 2016, A\&A, 549, 63

Pollina G. et al., 2019, MNRAS, 487, 2836

Postman M., Lubin L. M., Gunn J. E., Oke J. B., Hoessel J. G., Schneider D. P., Christensen J. A., 1996, AJ, 111, 615

Ramella M., Nonino M., Boschin W., Fadda D., 1999, in Giuricin G., Mezzetti M., Salucci P., eds, ASP Conf. Ser. Vol. 176, Observational Cosmology: The Development of Galaxy Systems. Astron. Soc. Pac., San Francisco. p. 108 ,

Rykoff E. S. et al., 2012, ApJ, 746, 178

Rykoff E. S. et al., 2014, ApJ, 785, 104

Rykoff E. S. et al., 2016, ApJS, 224, 1

Rykoff E. S., Rozo E., Keisler R., 2015, preprint (arXiv:1509.00870)

Saro A. et al., 2015, MNRAS, 454, 2305

Saro A. et al., 2017, MNRAS, 468, 3347

Sevilla-Noarbe I. et al., 2018, MNRAS, 481, 5451

Shectman S. A., 1985, ApJS, 57, 77 
Shipp N. et al., 2018, ApJ, 862, 114

Soares-Santos M. et al., 2011, ApJ, 727, 45

Starck J.-L., Murtagh F. D., Bijaoui A., 1998, Image Processing and Data Analysis, Cambridge University Press, Cambridge, UK,

Strazzullo V. et al., 2019, A\&A, 622, A117

Suchyta E. et al., 2016, MNRAS, 457, 786

Trevese D., Castellano M., Fontana A., Giallongo E., 2007, A\&A, 463, 853

Troxel M. A. et al., 2018, Phys. Rev. D, 98, 043528

Vikhlinin A. et al., 2009, ApJ, 692, 1060

Weinberg D. H., Mortonson M. J., Eisenstein D. J., Hirata C., Riess A. G., Rozo E., 2013, Phys. Rep., 530, 87

Wen Z. L., Han J. L., Liu F. S., 2012, ApJS, 199, 34

Wetzel A. R., Tinker J. L., Conroy C., van den Bosch F. C., 2013, MNRAS, 432,336

Yuan F. et al., 2015, MNRAS, 452, 3047

Zhang Y. et al., 2016, ApJ, 816, 98

Zhang Y. et al., 2017, preprint (arXiv:1710.05908)

Zhang Y. et al., 2019, MNRAS, 487, 2578

\section{SUPPORTING INFORMATION}

Supplementary data are available at https://www.linea.gov.br/catal ogs/wazp/ online.

Please note: Oxford University Press is not responsible for the content or functionality of any supporting materials supplied by the authors. Any queries (other than missing material) should be directed to the corresponding author for the article.

\footnotetext{
${ }^{1}$ Laboratório Interinstitucional de e-Astronomia - LIneA, Rua Gal. José Cristino 77, Rio de Janeiro, RJ 20921-400, Brazil

${ }^{2}$ Laboratoire d'Annecy De Physique Des Particules (LAPP), 9 Chemin de Bellevue, F-74940 Annecy, France

${ }^{3}$ Université Côte d'Azur, OCA, CNRS, Lagrange, UMR 7293, CS 34229,

F-06304 Nice Cedex 4, France

${ }^{4}$ Observatório Nacional, Rua Gal. José Cristino 77, Rio de Janeiro, RJ 20921 400, Brazil

${ }^{5}$ Departamento de Física Matemática, Instituto de Física, Universidade de São Paulo, CP 66318, São Paulo, SP 05314-970, Brazil

${ }^{6}$ Fermi National Accelerator Laboratory, P. O. Box 500, Batavia, IL 60510, USA

${ }^{7}$ Instituto de Fisica Teorica UAM/CSIC, Universidad Autonoma de Madrid, E-28049 Madrid, Spain

${ }^{8}$ Institute of Cosmology and Gravitation, University of Portsmouth, Portsmouth PO1 3FX, UK

${ }^{9}$ CNRS, UMR 7095, Institut d'Astrophysique de Paris, F-75014 Paris, France

${ }^{10}$ Sorbonne Universités, UPMC Univ Paris 06, UMR 7095, Institut d'Astrophysique de Paris, F-75014 Paris, France

${ }^{11}$ Department of Physics and Astronomy, Pevensey Building, University of Sussex, Brighton BN1 9QH, UK

${ }^{12}$ Department of Physics \& Astronomy, University College London, Gower Street, London WC1E 6BT, UK

${ }^{13}$ Instituto de Astrofisica de Canarias, E-38205 La Laguna, Tenerife, Spain

${ }^{14}$ Universidad de La Laguna, Dpto. Astrofísica, E-38206 La Laguna, Tenerife, Spain
}

${ }^{15}$ Department of Astronomy, University of Illinois at Urbana-Champaign, 1002 W. Green Street, Urbana, IL 61801, USA

${ }^{16}$ National Center for Supercomputing Applications, 1205 West Clark St., Urbana, IL 61801, USA

${ }^{17}$ Institut de Física d'Altes Energies (IFAE), The Barcelona Institute of Science and Technology, Campus UAB, E-08193 Bellaterra (Barcelona) Spain

${ }^{18}$ INAF-Osservatorio Astronomico di Trieste, via G. B. Tiepolo 11, I-34143

Trieste, Italy

${ }^{19}$ Institute for Fundamental Physics of the Universe, Via Beirut 2, I-34014 Trieste, Italy

${ }^{20}$ Centro de Investigaciones Energéticas, Medioambientales y Tecnológicas (CIEMAT), E-28040 Madrid, Spain

${ }^{21}$ Department of Physics, IIT Hyderabad, Kandi, Telangana 502285, India

${ }^{22}$ Santa Cruz Institute for Particle Physics, Santa Cruz, CA 95064, USA

${ }^{23}$ Department of Astronomy, University of Michigan, Ann Arbor, MI 48109, USA

${ }^{24}$ Department of Physics, University of Michigan, Ann Arbor, MI 48109, USA

${ }^{25}$ Institute of Theoretical Astrophysics, University of Oslo, P.O. Box 1029 Blindern, NO-0315 Oslo, Norway

${ }^{26}$ Jet Propulsion Laboratory, California Institute of Technology, 4800 Oak Grove Dr., Pasadena, CA 91109, USA

${ }^{27}$ Institut d'Estudis Espacials de Catalunya (IEEC), E-08034 Barcelona, Spain

${ }^{28}$ Institute of Space Sciences (ICE, CSIC), Campus UAB, Carrer de Can Magrans, s/n, E-08193 Barcelona, Spain

${ }^{29}$ Kavli Institute for Cosmological Physics, University of Chicago, Chicago, IL 60637, USA

${ }^{30}$ School of Mathematics and Physics, University of Queensland, Brisbane, QLD 4072, Australia

${ }^{31}$ Center for Cosmology and Astro-Particle Physics, The Ohio State University, Columbus, $\mathrm{OH} 43210$, USA

${ }^{32}$ Department of Physics, The Ohio State University, Columbus, OH 43210, USA

${ }^{33}$ Center for Astrophysics, Harvard and Smithsonian, 60 Garden Street, Cambridge, MA 02138, USA

${ }^{34}$ Australian Astronomical Optics, Macquarie University, North Ryde, NSW 2113, Australia

${ }^{35}$ Lowell Observatory, 1400 Mars Hill Rd, Flagstaff, AZ 86001, USA

${ }^{36}$ Department of Astrophysical Sciences, Princeton University, Peyton Hall, Princeton, NJ 08544, USA

${ }^{37}$ Institució Catalana de Recerca i Estudis Avançats, E-08010 Barcelona, Spain

${ }^{38}$ Physics Department, 2320 Chamberlin Hall, University of WisconsinMadison, 1150 University Avenue Madison, WI 53706-1390, USA

${ }^{39}$ Institute of Astronomy, University of Cambridge, Madingley Road, Cambridge $C B 3$ OHA, UK

${ }^{40}$ Instituto de Física, UFRGS, Caixa Postal 15051, Porto Alegre, RS $91501-$ 970, Brazil

${ }^{41}$ School of Physics and Astronomy, University of Southampton, Southampton SO17 1BJ, UK

${ }^{42}$ Computer Science and Mathematics Division, Oak Ridge National Laboratory, Oak Ridge, TN 37831, USA

${ }^{43}$ Department of Physics, Stanford University, 382 Via Pueblo Mall, Stanford, CA 94305, USA

This paper has been typeset from a $\mathrm{T}_{\mathrm{E}} \mathrm{X} / \mathrm{L} \mathrm{TE} \mathrm{X}$ file prepared by the author 